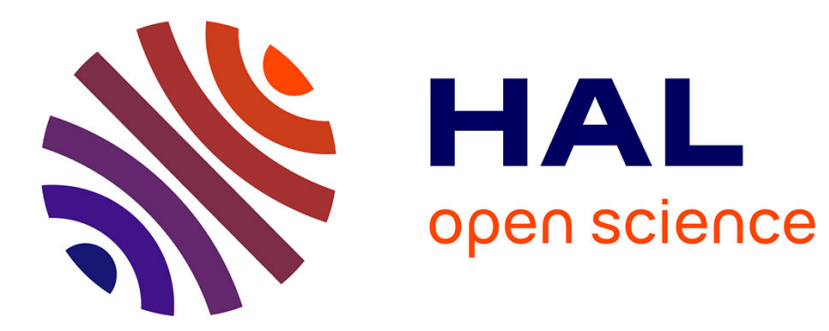

\title{
OR problems related to Home Health Care: A review of relevant routing and scheduling problems
}

Mohamed Cissé, Semih Yalçindag, Yannick Kergosien, Evren Sahin, Christophe Lenté, Andrea Matta

\section{- To cite this version:}

Mohamed Cissé, Semih Yalçindag, Yannick Kergosien, Evren Sahin, Christophe Lenté, et al.. OR problems related to Home Health Care: A review of relevant routing and scheduling problems. Operations Research for Health Care, 2017, 13-14, p.1-22. 10.1016/j.orhc.2017.06.001 . hal-01736714

\author{
HAL Id: hal-01736714 \\ https://hal.science/hal-01736714
}

Submitted on 13 Feb 2022

HAL is a multi-disciplinary open access archive for the deposit and dissemination of scientific research documents, whether they are published or not. The documents may come from teaching and research institutions in France or abroad, or from public or private research centers.
L'archive ouverte pluridisciplinaire $\mathbf{H A L}$, est destinée au dépôt et à la diffusion de documents scientifiques de niveau recherche, publiés ou non, émanant des établissements d'enseignement et de recherche français ou étrangers, des laboratoires publics ou privés. 


\title{
OR problems related to Home Health Care: A review of relevant routing and scheduling problems
}

\author{
Mohamed Cisséa $^{\mathrm{a}}$, Semih Yalçındağ ${ }^{\mathrm{b}}$, Yannick Kergosien ${ }^{\mathrm{a}}$, Evren Şahin ${ }^{\mathrm{c}}$, Christophe Lentéa ${ }^{\text {, Andrea }}$ \\ Matta $^{\mathrm{d}}$ \\ ${ }^{a}$ Université François-Rabelais de Tours, CNRS, LI EA 6300, OC ERL CNRS 6305, Tours, France \\ ${ }^{b}$ Industrial and Systems Engineering Department, Yeditepe University, 26 A ğustos Yerleşimi, Istanbul, Turkey \\ ${ }^{c}$ Laboratoire Génie Industriel, CentraleSupélec, Grande Voie des Vignes, Chatenay-Malabry Cedex, France \\ ${ }^{d}$ Dipartimento di Meccanica, Politecnico di Milano, Via La Masa 1, Milan, Italy
}

\begin{abstract}
The home health care routing and scheduling problem (HHCRSP) consists of designing a set of routes used by care workers to provide care to patients who live in the same geographic area and who must be treated at home. Hence, care activities, i.e., patient visits, must be planned to minimize measures, such as travel costs or to maximize the quality of service delivered to patients while respecting several constraints.

The HHCRSP is an extension of the vehicle routing problem (VRP) with unusual side-constraints that make the issues difficult to solve. This paper details a comprehensive overview of recent OR models developed for the HHCRSP, a field that has received a great amount of attention in recent years. To summarize the existing research contributions, we initially identify the most relevant features considered in the HHCRSP models, and then analyze the existing literature according to the way the different studies formulate the constraints and objective functions. We then provide an overview of methods developed to solve the HHCRSP and discuss future research directions.
\end{abstract}

Keywords: home health care, vehicle routing problem, literature review

\section{Introduction}

Home health care (HHC) consists of a wide range of health care services that are provided in patients' homes in cases of illness or injury. As reported in recent demographic studies, health care services in many countries are shifting from hospitals to home health care providers. According to Genet et al., 2013, in Europe $1 \%$ to $5 \%$ of the total public health budget is spent on $\mathrm{HHC}$, which is expected to generate three main advantages: a decrease in hospital admissions, a decrease in hospitalization duration, and ability for to remain in their homes. Recent literature reviews regarding operations research (OR) models applied to HHC can be found in Bashir et al., 2012, Benzarti et al., 2010, Gutiérrez and Vidal, 2013] and Bennett

Email addresses: mohamed.cisse@univ-tours.fr ( Mohamed Cissé ), semih.yalcindag@yeditepe.edu.tr ( Semih Yalçındağ ), yannick.kergosien@univ-tours.fr (Yannick Kergosien ), evren.sahin@ecp.fr (Evren Şahin ), christophe.lente@univ-tours.fr ( Christophe Lenté), andrea.matta@polimi.it (Andrea Matta) 
Milburn, 2012]. Additionally, a hierarchy of operations management decisions in HHC organizations has been recently proposed in [Matta et al., 2014, Sahin and Matta, 2015].

These studies reveal a great number of decisions related to the human resource planning issue that lead to OR problems (see Figure 1). Among these issues, the districting problem is a subject of interest at a strategic level. This problem consists of partitioning the HHC service territory into clusters of patients and aggregately assigning each cluster to a set of resources, e.g., care worker Benzarti, 2012, Blais et al., 2003. Districts are designed according to various criteria. For example, a first criterion can be the respect of the maximum distance between two patients assigned to the same district, which would be closer than the current acceptable value. Another possible criterion is the balancing of workloads among the designed districts. Resource dimensioning, another OR problem in HHC, addresses the problem of identifying the level of resources required in HHC and assigning these resources to the districts. Resources can be people or materials, e.g., the number of care workers to deliver care services or the number of technical devices required Busby and Carter, 2006. Resource dimensioning is generally done at a tactical level. At an operational level, two main problems appear: the operator assignment problem and the home health care routing and scheduling problem (HHCRSP). The first consists of deciding which care workers deliver care services to which patients Lanzarone et al. 2012, whereas the HHCRSP aims at scheduling patient visits assigned to each care worker. These two problems can be considered simultaneously or independently Yalcindag et al. $2016 \mathrm{ab}$.

Figure 1 summarizes the human resource planning process described above, by referring to the outputs associated with each decision level and the related OR problems.

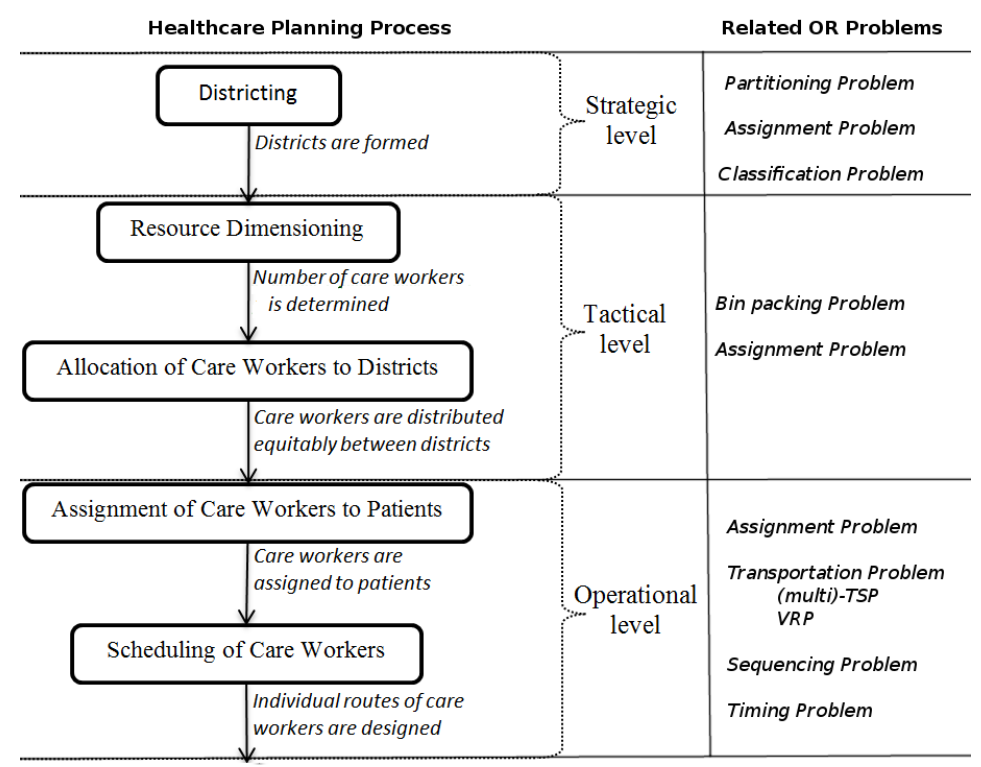

Figure 1: Human Resource Planning Process 


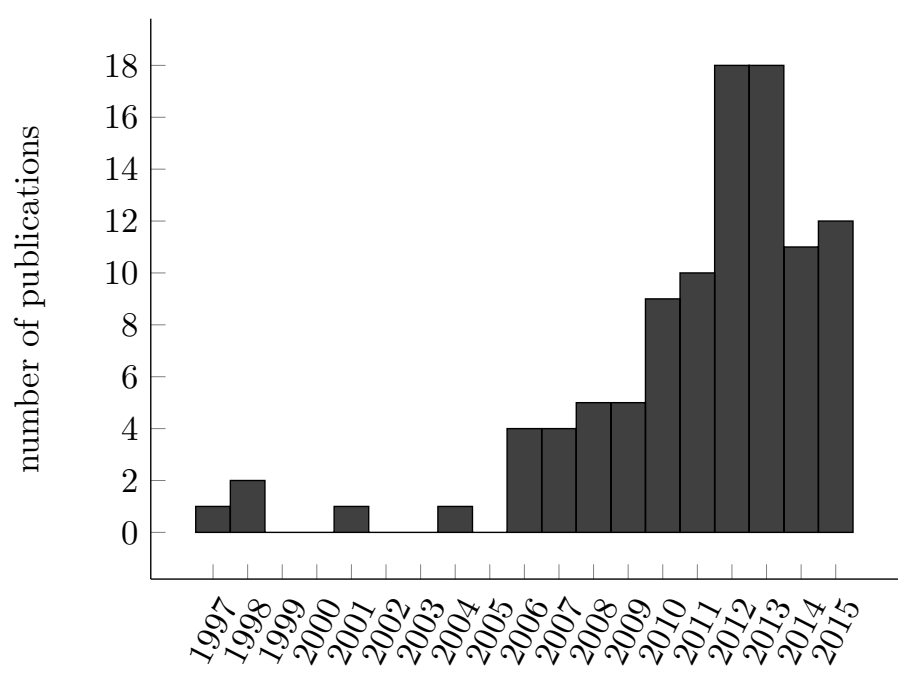

Figure 2: Publications in journals and conference proceedings by year

The goal of this paper is to analyze the existing literature pertaining to OR models applied to the HHCRSP that are found in relevant journal papers, conference proceedings, Ph.D. theses, and books that represent 17 years of work in this field. HHC routing and scheduling solutions differ from existing models since problems considered often originate from different national and regulatory settings. Existing publications were initially selected by browsing the bibliographic databases Web of Science, Science Direct, Jstor, semanticscholar, CiteSeerX and using Google Scholar. Keywords used in the search were: "home health care", "home care", "resource scheduling", "routing", and "vehicle routing problem". Additional publications were identified based on the reference lists of the selected works.

The present literature review differs from earlier studies because it focuses primarily on the HHCRSP on which an increasing number of academic studies have been conducted in the last two decades (Figure 22. This trend is due first to the fact that HHC is a promising and growing sector Bennett Milburn, 2012 and, second, most home care providers do not use OR tools to design care worker routes which can result in sub-optimal solutions. Furthermore, in most settings, the personnel dedicated to this task have limited skills because typically they come from a medical, rather than an OR background. Rintala et al., 2016 studied the potential role of operations management research in organizing and improving home care for the elderly. They presented a review of existing service operations management literature in order to present typical managerial implications. Next, they described a case study in a home care context, and conducted interviews. Working groups collected suggestions on the types of operational level problems the management research could solve and HHCRSP was identified among these problems.

A recent literature review related to HHC routing and scheduling problem was published Fikar and Hirsch, 2017. However, we propose a complementary and extended literature review with some important 
differences. First, Fikar and Hirsch, 2017] included mainly published journal articles available online before October 2015. We extended the review to other relevant sources of information, such as conference proceedings, book chapters and technical reports. We also extended the review to works dealing with the more general issue of routing and scheduling problems, which may concern both human resources as well as material resources, e.g., delivery of medicine or equipment. Moreover, features related to HHC operations are discussed in more detail and the studies are positioned in a larger framework that deals with OR problems in HHC. For example, the continuity of care that is a main feature in home care context, is integrated in different ways in existing models (e.g. as a constraint that states all visits of a patient is assigned to the same care worker or to a limited group, or as an objective function to minimize the number of different care workers per patient). In this case, we discuss how this feature and its different levels (full or partial continuity of care) is taken into account as assignment constraint or objective function in the literature. Thus, we propose a finer classification of the available literature, which is based on constraints in existing models and objective functions. Finally, we propose a deeper analysis of methods developed thus far to solve HHC routing and scheduling problems and a benchmark for instances and data sets used in the available literature. Two other literature reviews related to HHC routing and scheduling problem are available: a technical report Yalcindag et al. 2012a, which deals with human resource scheduling and routing problems in a home health care context, and a conference paper Mutingi and Mbohwa, 2013, which focuses on HHC staff scheduling. Papers included in the present paper are partially found in Fikar and Hirsch, 2017. Hence, our paper verifies some of the existing classifications in the literature and extends the topic by developing a more comprehensive and up-to-date review on the HHCRSP.

To classify existing research contributions, we propose a review that highlights the most relevant features, constraints and objectives considered in the existing studies. We also present available methods to solve the HHCRSP and provide directions for future research.

This paper is organized as follows: In Section 2 , the HHCRSP is briefly described by highlighting relationships with some well-known combinatorial optimization problems. Section 3 describes features that we consider the most relevant for the HHCRSP models and details how they are modeled as constraints

in the literature. In Section 4 existing papers are classified according to how they formulate the objective functions. Then, Section 5 addresses methods that enable obtaining exact and approximate solutions for the HHCRSP. Section 6 provides a discussion on results obtained and concludes with suggesting for promising future research directions.

\section{Home health care routing and scheduling problem}

The vehicle routing problem (VRP) Toth and Vigo, 2001, Laporte, 2007 is a widely studied combinatorial optimization problem that occurs in many applications, for example in gasoline delivery Dantzig and Ramser. 


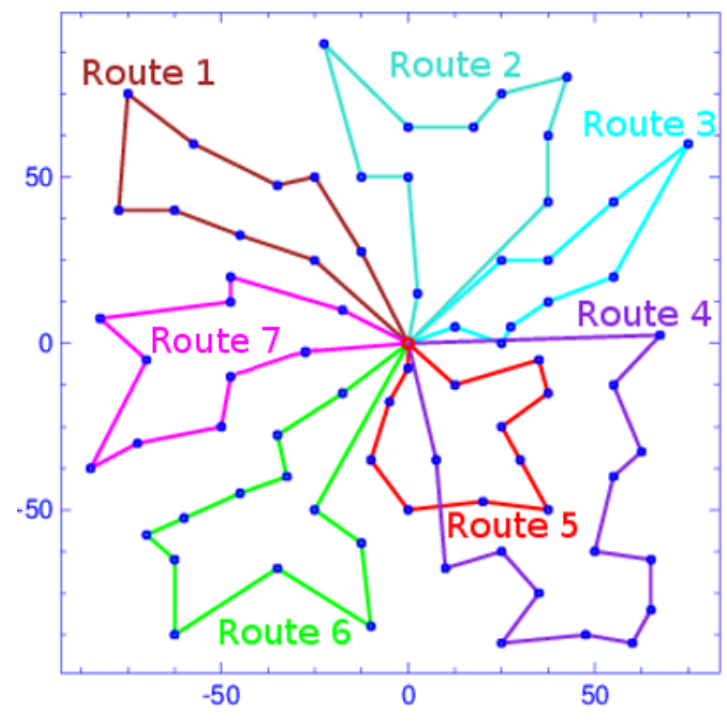

Figure 3: A VRP solution with 1 depot, 75 vertices and 7 routes

1959], newspaper distribution Toth and Vigo, 2001, and food delivery Russell, 1995].

VRP is generally defined as follows: Let $G=(V, A)$ a graph with $V$ the set of vertices, and $A$, the set of arcs. The set of vertices represents customers to serve and a single depot from which each vehicle route must begin and end. The decision maker must design a set of routes that minimizes the total traveling cost such that every vertex is visited once (Figure 3 represents a possible solution of the VRP). Since the work of Dantzig and Ramser, 1959, many extensions of the VRP have been studied. One of the most well known extensions is the vehicle routing problem with time windows (VRPTW) Bräysy and Gendreau, 2005a b]. In this version, each customer $i$ must be visited within the time interval $\left[e_{i}, l_{i}\right]$ where $e_{i}$ and $l_{i}$ are the earliest and latest times to start a visit, respectively.

The HHCRSP is an extension of VRP augmented by many unusual side-constraints that are specific to the HHC context. In the literature, HHCRSP is described in different terms: home health care scheduling problem Begur et al. 1997, home health care routing and scheduling problem Mankowska et al., 2014, home health care problem Bertels and Fahle, 2006, home care crew scheduling problem Rasmussen et al. 2012, home care worker scheduling [Akjiratikarl et al., 2007, home help staff scheduling Ikegami and Uno, 2007 and therapist routing and scheduling problem Bard et al., 2014. According to several authors Trautsamwieser and Hirsch, 2011, Hiermann et al., 2013, Di Gaspero and Urli, 2014, the first studies on the HHCRSP date back to 1997 Begur et al. 1997] and 1998 [Cheng and Rich, 1998.

The HHCRSP can be described as follows: a set of patients scattered in a geographic area who need care services, i.e., visits at home, which must be provided by care workers. An extended definition of HHC is used, which includes various health related services performed at clients' homes such as home care, mobile care, and extramural health care, terms that are often used interchangeably in the literature. The problem 
consists of designing a set of routes to provide the planned care visits over a planning horizon that minimizes criteria such as cost or maximizes the quality of service while respecting several constraints. This problem is similar to a VRP because the main decision is to design a set of routes that serves all patients. However, studies addressing the HHCRSP often differ from VRP because of the presence of specific features that generate new constraints that are more challenging to consider. The following features make the problem more challenging to solve:

- Continuity of care ensures that each patient is assigned to a restricted set of care workers. When this set is reduced to a unique care worker for all visits of a given patient over the planning horizon, the HHC provider is said to operate under "full continuity of care".

- Temporal dependency and disjunctive services are care services can have relationships among them. For example, one service should be planned three days after another service, or two services cannot be provided to a patient at the same time.

- Care worker and patient characteristics are considered because professionals have different areas of expertise, which generate qualification/skill constraints in assignment decisions. Patients may also have requests that the planner should handle, e.g., preferences for a care worker, gender, language, etc.

Because of the first two features, the decision to assign a care service to a worker cannot be done without considering interactions with the other care services. The last feature increases the complexity of the assignment and timing problems of the HHCRSP. These features will be described in more detail in Section 3.

One of the main characteristics of the existing HHCRSP literature is the diversity of models, which might be explained by the variety of features and by the modeling choices regarding the problem formulation. Most of the HHCRSP models are formulated as VRP or extensions of VRP. These models include, the vehicle routing problem Toth and Vigo, 2001, vehicle routing problem with time windows (VRPTW) Bräysy and Gendreau, 2005b a , multiple depot traveling salesman problem with time windows (MDTSPTW) Bektas, 2006], periodic vehicle routing problem (PVRP) Francis et al. 2008 and periodic vehicle routing problem with time windows (PVRPTW) Francis et al. 2008. Other studies include models based on the set partitioning problem Bredstrom and Ronnqvist, 2007, Eveborn et al., 2006, 2009, Rasmussen et al., 2012.

\section{Features considered in the HHCRSP models}

Features considered in the existing HHCRSP models reflect the diversity of HHC operations. Hence, OR modelers working in this area have developed a large set of modeling choices that depend on features integrated into the problem formulation. Our literature reveals that those features can be divided into three groups depending on whether they concern the HHC service organization, patients, or care workers (cf. Table 
1). A given HHCRSP model results from the combination of features considered in the formulation of the problem. Each feature is formulated as either a constraint presented in this section or a satisfaction criterion in the objective function presented in Section 4 .

For each feature, constraints can also be grouped according to their nature (cf. Table 1). Three classes of constraints have been identified: temporal constraints, assignment constraints and geographic constraints. The first group concerns constraints that are associated with a time relationship or a frequency-over-time relationship, i.e., at what time the $\mathrm{HHC}$ service can start or when a care worker can provide a service according to its time schedule. Assignment constraints are largely related to the relationship between care workers and patients without considering the time aspect. They have a direct impact on the identification of the care worker who can provide a service to a given patient. Finally, the last group of constraints concerns geographical aspects, i.e., location and type of network flow. This section reviews existing papers according to how problem features are modeled as constraints. At the end of the section, Tables 24 provide a detailed overview of journal papers with respect to the planning horizon, objective function, and the main constraints.

\begin{tabular}{|c|c|c|c|}
\hline Actors & Temporal constraints & Assignment constraints & Geographic constraints \\
\hline $\begin{array}{l}\text { HHC service } \\
\text { organization }\end{array}$ & $\begin{array}{l}\text { - Planning horizon } \\
\text { - Frequency of decision }\end{array}$ & - Continuity of care & $\begin{array}{l}\text { - Sectors/districts } \\
\text { - Typology of HHC } \\
\text { services provided }\end{array}$ \\
\hline Patient & $\begin{array}{l}\text { - Frequency of visits } \\
\text { - Time windows } \\
\text { - Temporal dependency } \\
\text { - Disjunctive services }\end{array}$ & - Preferences & $\begin{array}{l}\text { - Type of network } \\
\text { between home locations }\end{array}$ \\
\hline Care worker & $\begin{array}{l}\text { - Contract type } \\
\text { - Capacity/working } \\
\text { hours }\end{array}$ & $\begin{array}{l}\text { - Qualification/skill } \\
\text { - Workload balancing }\end{array}$ & - Location of care workers \\
\hline
\end{tabular}

Table 1: Classification scheme based on constraints

\subsection{Constraints related to the HHC organization}

\section{Temporal constraints}

The "planning horizon" used in the routing decision refers to the period for which the HHC provider plans the assignment and routing decisions. The length of the planning horizon depends on the availability and quality of information available. Indeed, in the first periods of the horizon, most information used for 
planning will be accurate, whereas the planner must work with forecasts for subsequent periods. The planning horizon considered in the HHCRSP models is often one day Akjiratikarl et al., 2007, Bredström and Rönnqvist, 2008, Bertels and Fahle, 2006, Liu et al., 2013, Mankowska et al., 2014, Rasmussen et al., 2012, Trautsamwieser et al., 2011] or one week Bard et al., 2014, Liu et al., 2014, Trautsamwieser and Hirsch. 2014. In Wirnitzer et al. 2016, a planning horizon of four weeks has been considered. Note that most papers consider a planning horizon of one week or less, generally extending the horizon on a rolling basis (cf. Bennett and Erera, 2011). The planning horizon is extended to two to three months by Hewitt et al. 2016. who then quantify the advantages of considering a longer time frame while planning. For this case, they compare two nurse-to-patient assignment strategies: a rolling weekly strategy and a long term planning strategy where planning is done once for the entire horizon (two to three months).

The "frequency of routing decision" refers to how often the routing decision is repeated within the planning horizon. Updates in the routing decisions largely stem from information updates pertaining to patients, care workers, and more generally system status, e.g., evolving patient and care worker availability. The reasons are that new patients continually enter the system and conditions of previously admitted patients and care worker availabilities change. Therefore, either routing decisions can be modified at fixed time intervals or the update can be made when certain conditions are met. The frequency of routing decisions used in the literature is either for every sub-period within the entire planning horizon Mankowska et al. 2014 or only once at the beginning of the planning horizon Bard et al. 2013.

\section{Assignment constraints}

The assignment constraints of an HHC service organization are largely related to the continuity of care, which is also called patient-nurse loyalty in Nickel et al. 2012 or employee regularity Gamst and Jensen, 2012. The notion of continuity of care constraint considered in the HHCRSP models is similar to the "person-oriented consistency" in PVRP literature Kovacs et al., 2014, also called the consistent vehicle routing problem (ConVRP), in which customers must be visited by the same deliverer and during approximately the same time. According to quality standards adopted by HHC providers, care services to patients can be provided under full, partial or no continuity of care. Under full continuity of care, the HHC provider assigns a patient to one and only one care worker, who is responsible for that patient's care during his/her stay in the HHC service. Such an organization enables the prevention of losses of information among care workers, and reinforces the relationship between the patient and the care worker. Indeed, according to Freeman and Hughes, 2010, keeping the same care worker builds a relationship of confidence between the

patient and care worker. Such a relationship can be important in contexts such as maternity Bowers et al. 2015], in which antenatal and postnatal care of a given mother are performed by the same midwife. Partial continuity of care is usually observed in situations in which a patient requires more than one type of care. 
If one of the care types is more frequent, e.g., more than $50 \%$ of the total care required, then a reference care worker can also be assigned (as in the full continuity of care case) to coordinate the required services delivered by other care workers. In the no continuity of care case, the provider does not need to respect the care worker-patient assignment information coming from a previous period. Each new period starts with a new assignment process, in which all available care workers can be assigned to all patients according to their skills and patient requirements. In most cases, it is difficult to keep the same care worker for the same patient while respecting temporal and assignment constraints. Thus, this constraint is generally considered as a soft constraint as seen in Macdonald et al. 2009. However, Hewitt et al., 2016] consider full continuity of care as a hard constraint. Full and partial continuity of care can be mixed [Duque et al., 2015], where the same care worker is assigned to all visits for a patient that requires one or two visits per week. Otherwise (when there are more than two visits per week), at least two care workers should be assigned to all visits over the entire planning horizon. Finally, in Wirnitzer et al., 2016, patients can be visited up to three times per day. Due to this, ensuring full continuity of care with a single care worker is not usually possible. Thus, they focus on keeping the number of care workers assigned to each patient at the minimum level.

\section{Geographic constraints}

In terms of team organization and management, the HHC provider can decide to cluster the existing teams in districts according to relevant criteria such as territory, skills of care workers and requirements of patients. Such a districting approach enables reducing care workers' travel times (inside a given district) and forming smaller care teams that can be easier to manage. The single-district case considered by Mankowska et al. 2014 is the simplest alternative, in which the provider does not split workers into smaller clusters. In the multiple-district case considered by Eveborn et al. 2006, each district can be managed independently as an autonomous decision center Duque et al. 2015 or in an integrated fashion Lanzarone et al. 2012. In the multiple-district case, a care worker is assigned to a geographical area and may only serve patients within that area or a subset of areas. This type of restrictions can be modeled as a skill constraint.

Different types of HHC services have been modeled in the literature. In addition to classical care services, other types of services are considered by Bräysy et al., 2009, Liu et al., 2013, 2014, who address the problem of collecting biological samples and/or delivery of drugs or materials. In terms of modeling, these problems are similar to VRP with pickup and delivery [Toth and Vigo, 2001, Chapter 9]. HHC is thus a decentralized care system in which the location of entities that participate in the care delivery process has an impact on routing decisions. The location of the HHC center, medical laboratories, pharmacies and chemotherapy/radiotherapy centers involved in the care provided are among the parameters to be considered in the HHCRSP models. Therefore, routes are optimized considering these locations. For example, Kergosien et al. 2014 propose a model to schedule blood collection at home. In this context, some stops at laboratories 
should be planned in the route because blood samples have an expiration time. To consider the stop at a laboratory, Elbenani et al. 2008 introduced the notion of "fictitious destination nodes" that models the return to a laboratory after a blood sample is performed. Kergosien et al., 2014 consider timetables of analysis for each laboratory. In the case in which biological samples must be delivered, a constraint on maximum transportation time must be considered due to the limited lifetime of the product. The sample lifetime is particularly important in chemotherapy because of its perishability as underlined by Chahed et al. 2009. This type of constraint generates difficulties in building feasible routes because the deadline of the delivery time at the patient home depends on the time the product has been prepared or the time the care worker has left the center with the product. In the case of collecting biological samples, the deadline for returning samples to a laboratory depends on the time of the collection. The maximum transportation time constraint can be found in the literature on VRP for perishable food delivery because of its shelf life Amorim and Almada-Lobo, 2014. Bräysy et al. 2009 focus on home meal deliveries that consist of vehicles starting from a central kitchen to deliver meals to patients, and picking up and returning the packaging to the central unit if it can be reused. Liu et al. 2013, 2014 consider a VRP with delivery and pickup operations: transportation of drugs/medical devices between the HHC center and patient homes, delivery of special drugs from the hospital to patients, and delivery of blood samples from patients to a laboratory.

\subsection{Constraints related to patients}

\section{Temporal constraints}

When patients are admitted to the system, the HHC provider explains the therapeutic project (ThP), which is used to specify the nature and frequency of visits required by the patient. Based on the ThP, a category, namely a care profile, is assigned to the patient based on the types of visits required, i.e., the required care worker type and qualifications and care intensity. Care intensity, for example could involve the volume of care needed for palliative and non-palliative patients. The care profile also defines the frequency of visits, e.g., every day or once a week. In the literature, in terms of constraints on the frequency of visits, some studies consider that a patient should be served once per day Hiermann et al. 2013 or per week Bard et al. 2014. However, other studies allow a patient to be visited several times a day Rasmussen et al. 2012 or a week Nickel et al., 2012. In this case, the patient usually needs several services Kergosien et al., 2009.

Most studies assign a time window to each patient, i.e., a time interval in which the patient is available at home to receive care. Time windows can be hard/fixed Akjiratikarl et al., 2007, Bard et al., 2014, Liu et al. 2017, Redjem and Marcon, 2016 or soft/flexible Mankowska et al., 2014, Trautsamwieser and Hirsch. 2011, Hiermann et al. 2013, Nickel et al. 2012. In the first case, the decision maker is forced to schedule the visit within the time window. In the second case, time windows can be violated and delays can be accepted with a penalty cost. Bertels and Fahle, 2006 considered two types of time windows for each service; one is a soft time window that is included in a second one, a hard time window in which the service must begin. 
Other specific constraints on the starting time of service can be found in the literature. In Bennett, 2010, the starting time has been fixed to a value belonging to a set of "equally spaced appointment times". The sequence of numbers in this set can be defined as an arithmetic progression. Thus, the set of possible values within which a service can start is separated by an identical value. A similar approach has been adopted in Di Gaspero and Urli, 2014, Wang, 2011, Shao, 2011. Finally, only Braekers et al., 2016 have introduced both time window and preferred starting time.

Some HHCRSP studies assume a regular frequency of visits, which refers to the principle that a set of services for a given patient should occur at the same day and time of day each week, e.g., every Tuesday at $2 \mathrm{pm}$. This principle is also referenced in the literature as the cycle or the periodicity of the service. This type of constraint is considered by [Duque et al., 2015, Bennett and Erera, 2011, Bennett, 2010. In the two last works, a rolling horizon approach has been used to solve a dynamic periodic routing problem in which a weekly frequency over a prescribed number of weeks is respected. In addition, weekly visits must be repeated at the same days and times (with a visit-time consistency constraint) by using patterns of visits that repeat in different weeks. In Duque et al. 2015, each patient is assigned to a number of visits per week (one to five times a week) with the following constraint: patients cannot be visited on consecutive days (unless more than four visits are planned). In the VRP literature, the service frequency feature can be found in other problems, such as the waste collection problem Baptista et al., 2002.

In Wirnitzer et al. 2016, all patients that should be visited during a week (up to three times per day) are clustered and assigned to predefined master tours. A master tour is defined by a shift, a starting time, a duration and the set of patients belonging to this tour. All master tours in a week are referred to as the "master tour plan" that remains consistent from week to week. The problem consists in assigning care workers to the master tours.

In studies that consider a planning horizon of several days, patients are also characterized by a set of days where they can be visited. These days represent the availabilities or preferences of patients. A visit can only be scheduled on a day from this set. Because it is possible that care workers also have a set of unavailable days, the decision maker must find a match for the availabilities of both patients and care workers. This feature has been considered in a few studies Bard et al., 2014, 2013. In Hewitt et al., 2016, each patient is associated with a period of care (two to three months) and a weekly care plan that defines the days a patient is to be visited each week.

In some cases, the provider must also consider other constraints such as providing a minimum number of visits and/or imposing a time lag between visits. For example, a patient must be visited at least two times per week and/or at least two days between visits should be respected [Begur et al., 1997]. This situation involves defining a pattern assignment for each patient Bard et al., 2014, Cappanera and Scutellà, 2015, Liu et al. 2014]. Pattern assignments correspond to a subset of days in a period, e.g., a week, in which care services 
must be performed. This limited number of possible combinations of visiting days allows feasible scheduling from both providers' and patients' points of view. For example, a patient with two visit requirements can be visited on either Tuesday and Friday (pattern 1) or Monday and Thursday (pattern 2). Duque et al., 2015 have defined pattern assignments as time slots of four hours (half-days: morning or afternoon) in which the patient must be served. Each patient can provide his/her preferences concerning the time slots for weekly visits.

Visits assigned to a given care worker may have a temporal dependency. Dependencies can exist among the routes of different care workers or among the services provided by a care worker within the same route. In the first case (dependency among routes), visits may require more than one care worker at the same time to provide synchronized or shared services. For example, such a requirement arises when an overweight patient requires two persons to lift him/her and the routes of different care workers must be coordinated. Eveborn et al. 2006, Thomsen, 2006 considered synchronized/shared services. As shown in Thomsen. 2006], this is not a simple concept, and it becomes even more complicated when the capacity of care workers is limited, unlike in classic VRP, in which routes can be evaluated independently. Determining the best timing for a care worker's shared visits, and then his/her whole route, i.e., the starting time of each visit in the route, cannot be done independently from the other routes related to the shared visits. Figure 4 illustrates a case with four routes and three shared visits. If the route timing of $R 1$ is computed, it should be done with $R 2, R 3$ and $R 4$ routes. The shared visit constraint can be viewed as a specific case of temporal dependency between services for which the time between shared services is not necessarily equal to zero. The delay between shared services can be fixed Kergosien et al. 2009 or can be bound between a minimum and a maximum value Mankowska et al. 2014. For example, if drugs must be administered to a patient, a minimum time before providing a meal must be set. Models dealing with a minimum and maximum time between shared services lead to considering precedence constraints between services Bredström and Rönnqvist, 2008, Redjem and Marcon, 2016. Such constraints can be found in the rich VRP literature. Practical applications other than HHC can be found in transportation of disabled passengers at airports Reinhardt et al. 2013, or military aircraft mission-planning Quttineh et al., 2013 in which aircraft must be synchronized in both time and space during attacks. A survey of vehicle routing problems with multiple synchronization constraints and a classification of different types of synchronization can be found in Drexl, 2012 .

In the second case (dependency within routes), a certain visit must be conducted before another because clinical precedence relationships among services exists. In addition to temporal dependency, Kergosien et al. 2009 introduce the notion of disjunctive services, also called exclusion of services. Two disjunctive services must not be performed simultaneously. This situation refers to potential incompatibilities between services that cannot be provided to a patient at the same time. For example, physical therapy cannot be 


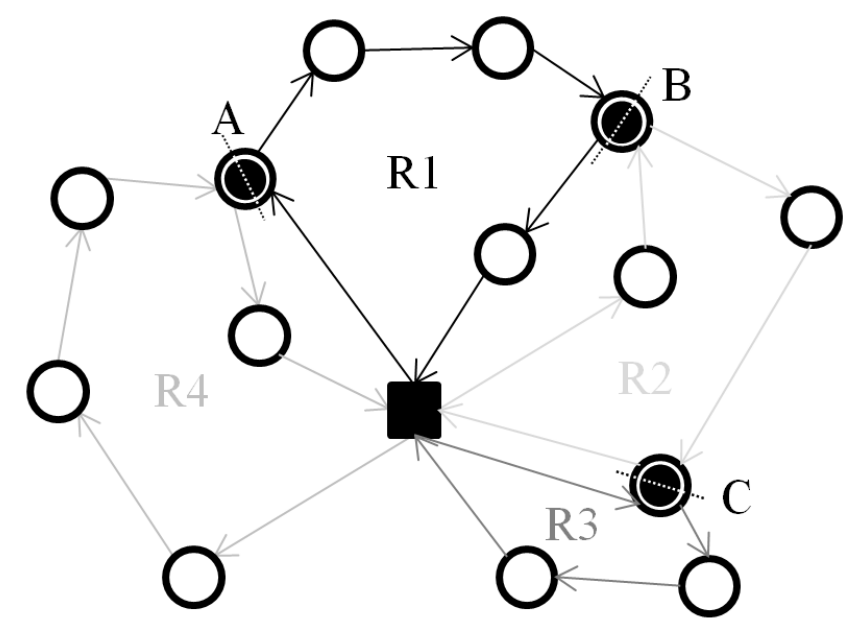

Figure 4: Example with four routes and three shared visits (A, B and C)

delivered at the same time that a blood sample is collected. This type of constraint adds an additional level of decision on sequencing the visits of care workers for each patient. In such a case, the sequence of patients for each care worker is no longer sufficient to encode a solution. Redjem and Marcon, 2016 considered all services assigned to the same patient as disjunctive. Synchronized/shared services, precedence and disjunctive services have also been studied in Rasmussen et al. 2012, in which the authors have presented temporal dependency in five ranges: synchronization, overlap, minimum difference, maximum difference, and minimum plus maximum difference.

\section{Assignment constraints}

Finally, in terms of patient preferences, some authors model the case of patients who may reject a care worker for gender incompatibility or personal reasons [Trautsamwieser et al., 2011, Wirnitzer et al., 2016.

\section{Geographic constraints}

The type of network is an important feature of the HHCRSP. Depending on the context (rural or metropolitan) and on where patient homes are scattered in the region, traveling times of care workers can be significant. Rest et al. 2012 have underlined the differences between rural and urban services. Indeed, the ratio between care and traveling duration would be different in these cases. Estimating travel times is also difficult. Although most studies use Euclidean distances for experiments, some studies compute travel times based on a geographic information system Begur et al., 1997. Few studies Rendl et al., 2012, Hiermann et al. 2013 have considered a multimodal transportation network. In these studies, three main modes of transportation are considered: car, walking and public transportation. Public transportation differs in that a time schedule should be used. Travel times between two patient homes can therefore change depending 
on that time. Finally, Rest and Hirsch, 2016 have proposed a model that considers time-dependent travel times because these can change considerably according to the time of day, e.g., rush hour or off-peak times.

\subsection{Constraints related to care workers}

\section{Temporal constraints}

The capacity of a care worker refers to the time frame in which she/he must serve the patient according to the type of contract. The care worker can be full time, half time or an externally subcontracted employee of the HHC provider. Overtime work refers to working hours exceeding the regular capacity of a care worker. In such a case, the HHC provider pays additional compensation for overtime hours. Availability refers to the period over which the care worker is able to reach homes and visit patients. It is obtained by excluding breaks, vacations and days off from the care worker's capacity.

Most HHCRSP studies assume that care workers have predefined working hours, which do not include overtime, during which they can provide care services to patients. Depending on the study, this time window can be hard Hiermann et al., 2013, Braekers et al. 2016, Liu et al., 2017, Redjem and Marcon, 2016 or soft Trautsamwieser et al. 2011] according to whether overtime is possible. In the case of a hard time window, a solution with overtime for care workers is considered infeasible. In the case of a soft time window, a violation of the time window, i.e., overtime, is penalized in the objective function. However, it is often impossible for the care worker to begin his service before the starting time of the time window. Bertels and Fahle, 2006 have considered both hard and soft time windows; soft time windows are included in hard time windows, thereby limiting the overtime of care workers. With a planning horizon of more than one day, the time window of a given care worker potentially depends on the day in which it is considered Bard et al. 2014.

In addition to time windows, one solution to constrain the daily workload of a care worker is to limit the only daily working time; the shift start time is not fixed (and, therefore, must be determined), and the worker cannot exceed the daily working time. This approach employs an additional variable, the starting time of the care worker. This additional determination of daily working limits is country-dependent and concerns legal provisions. For example, this limit corresponds to 7.5 hours in the UK according to Akjiratikarl et al. 2007 or 8 hours in Finland according to Bräysy and Gendreau, 2005b. When the daily working time is exceeded, every supplementary hour is considered as overtime Nickel et al. 2012]. Overtime is allowed, but each time unit is penalized with a higher cost in the objective function. This limit can also be set as a weekly availability Duque et al. 2015, in which each care worker is allowed to work a maximum number of hours per week depending on her/his type of labor contract. In Wirnitzer et al. 2016], care workers are characterized by a working time limit per period according to their contracts. This is in addition to a daily working time regulation. This limit can be exceeded up to a maximum overtime per period. Moreover, care workers are not allowed to work two subsequent weekends and they must have a legal minimal break of nine hours between the late night and early morning tours. 
In the case of a time window, the visit times of a route can be computed by considering that a care worker leaves the depot at the start time of the time window. However, in the limited working time case, the departure time of a care worker from the depot depends on the time windows of patients on the route. It may be advantageous to delay departure from the depot using, for example, cf. the forward time slack procedure Savelsbergh, 1992.

Care worker breaks are also considered as temporal constraints. Two types of constraints exist in the literature: forcing the care worker to take a break either within a specific time interval Trautsamwieser and Hirsch, 2011, Liu et al. 2017] or when a certain route length is reached Thomsen, 2006. The routes of the care worker must be designed to respect the break timing, if it is provided, and the break duration, which implies that starting times of the breaks are additional decision variables. In some studies, break constraints can be modeled by representing each break using a fictitious patient, e.g., Kergosien et al., 2009, or by adding an idle time between two patient visits, e.g., Bard et al. 2013 Liu et al., 2017. To satisfy features related to care workers' contracts, some studies have introduced multiple contract types. For example, some models distinguish full-time and part-time care workers Begur et al. 1997. They are not subject to the same constraints, and the cost of each type of contract is different.

Finally, in the case of a medium- or long-planning horizon, i.e., a week or month, the decision maker should consider the unavailability of care workers Bard et al. 2013, Nickel et al., 2012, Wirnitzer et al. 2016. In the literature, these unavailabilities are due to working-day preferences, holidays, days off or sick leaves.

\section{Assignment constraints}

The first type of assignment constraint that can be found in the literature concerns worker qualifications. Care workers must satisfy qualification requirements, also called skill requirements, to provide a specific service Mankowska et al. 2014, Braekers et al., 2016, Duque et al., 2015. The qualifications/skills can be related to the function of the care worker (such as doctor, physical therapist, or nurse), gender constraints, or language skills Eveborn et al. 2009. This type of constraint is often modeled in three different ways. First, a single qualification is associated with each care worker and each care is characterized by a required qualification. To provide care to a patient, the qualification must match the requirement (e.g., Kergosien et al. 2009]). If there is not a relationship between services, the routing problem can be decomposed into several independent routing problems, one for each qualification. The second approach is to assign several qualifications to each care worker rather than only one Bertels and Fahle, 2006, which is a more general solution. Finally, the last approach, which can also be modeled by the second approach, is based on the level of qualification. According to Cire and Hooker, 2012, each care worker should be associated with a level of qualification, and each service requires a qualification level that represents the minimum level for a care worker who should perform the service. More information about the skill vehicle routing problem can 
be found in Cappanera et al., 2011].

In some studies, care worker preferences must be considered because they affect assignment considerations. These studies support the fact that a care worker can reject a patient due to personal reasons Trautsamwieser et al., 2011, Trautsamwieser and Hirsch, 2011, or allergy reasons Rendl et al., 2012, Rest and Hirsch, 2016, Hiermann et al., 2013, Wirnitzer et al. 2016. In terms of modeling, this constraint is clearly close to qualification requirements. In Liu et al., 2017, it is also used to maintain the continuity of care: a subset of care workers is assigned to each patient.

The second type of assignment constraints found in the literature is related to workload balancing to guarantee equity among care workers Ikegami and Uno, 2007 and thus keep workers motivated and reduce turnover within an organization. Because a perfect balance of the workload is difficult to obtain, this constraint is generally embedded in the objective function and considered as a soft constraint. The evaluation of the workload of a care worker can be done through several indicators, such as the number of visited patients or used capacity. Obviously, the type of contract should also be considered in the evaluation of the workload.

\section{Geographic constraints}

Most studies consider a single HHC center from which each care worker's route must start and finish; this point is also called a depot in the vehicle routing problem. The case of multi-depots Akjiratikarl et al., 2007. is less frequently considered. If the service area is large and not easily accessible because of territorial aspects, i.e., urban or non-urban area, there can be more than one health care center. Alternatively, care workers can also start and finish their activities from their own homes. Trautsamwieser et al., 2011 introduced three types of care workers according to the starting and ending locations: those whose routes start and end at the hospital, those whose routes start and end at home, and those whose routes start and end at home but the route duration between the care worker's home and the patient are not considered in the working time. Some models Bard et al., 2014, Kergosien et al. 2009 are more generic, by dissociating the starting and ending depots for each care worker. Thus, they cover both single and multiple depots.

To conclude this section, Table 2, Table 3, and Table 4, present a detailed overview on existing works published in scientific journals. For each study, the planning horizon, objectives and main constraints are given (workload balancing is considered a constraint even if it is embedded in the objective function). Among the features presented in this section, those that are the most specific to the context of HHC are: patient preferences, continuity of care (also called person-oriented consistency in VRP literature), qualifications or skills of care workers, workload balancing between care workers, temporal dependency, and disjunctive services and multiple contract types. Figures 5 and 6 show which studies, among those published in journals and dealing with the HHCRSP, consider these features for short and long planning horizons. These figures 


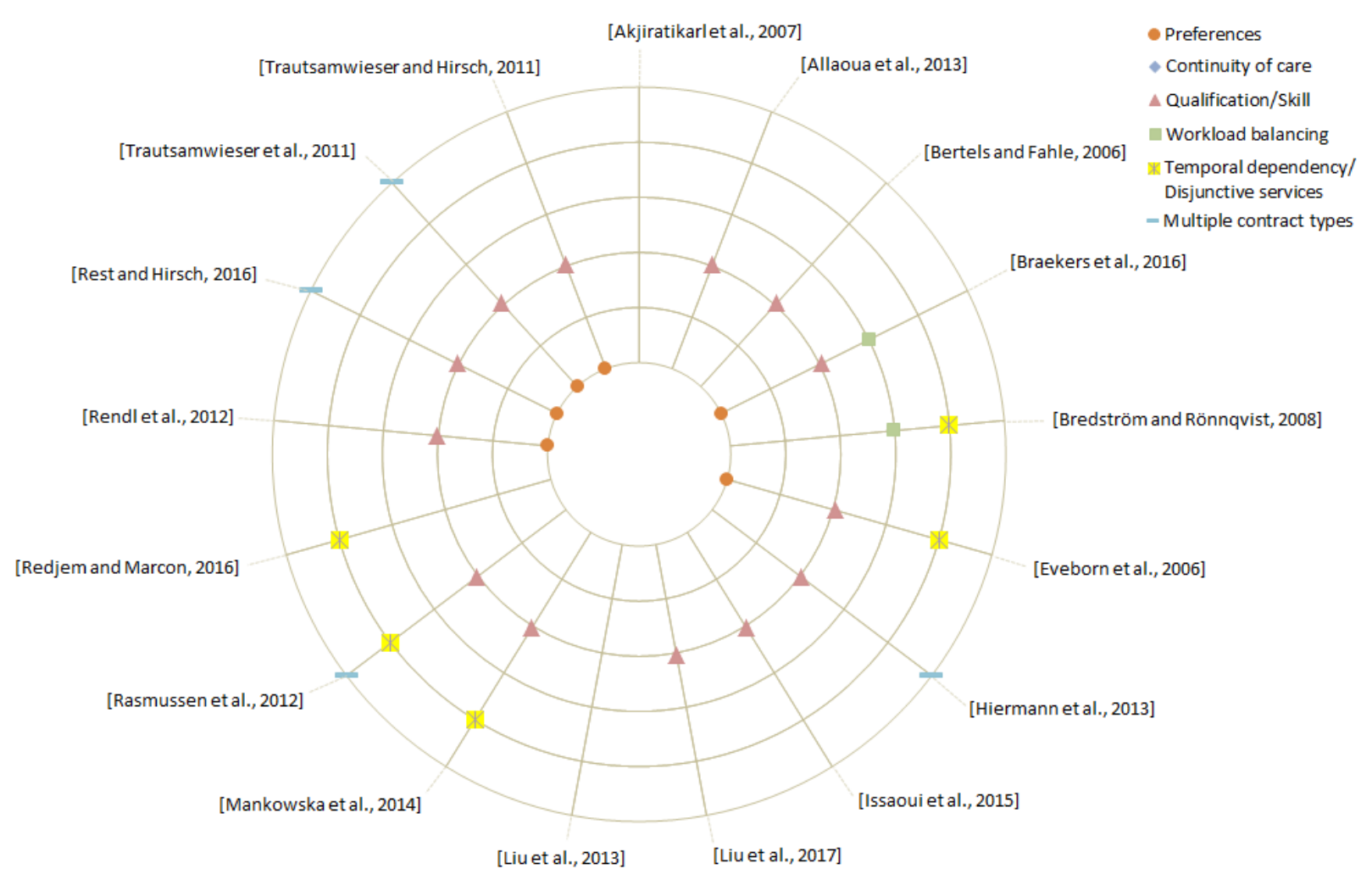

Figure 5: Publications with respect to some home care features - short planning horizon

show that the feature that is most considered in studies is the qualification or skill of care workers. On the other hand, the multiple contract types of care workers are less considered. The continuity of care is not considered in many studies, though it is a key service quality indicator. One of the drawbacks of short planning horizon studies is that they cannot easily take this figure into account. Finally, Figures 5 and 6 also show the lack of generic models since, at most, three of those features are considered at the same time.

\section{Objective Function}

In the literature, single-objective or multi-objective functions have been proposed to evaluate the quality of solutions. In the multi-objective case, the number of criteria varies from 2 Akjiratikarl et al., 2007 to 7 Trautsamwieser and Hirsch, 2011] to 13 Hiermann et al. 2013. In most studies, a weighted sum approach is used to evaluate solutions, even when the criteria do not have the same unit Bertels and Fahle, 2006. Steeg and Schröder, 2008, Mankowska et al. 2014. To the best of our knowledge, only Braekers et al., 2016] have proposed to enumerate the entire Pareto frontier using an $\epsilon$-constraint approach whereas Duque et al. 2015 have proposed a lexicographic approach.

In this Section, we present the most frequently used criteria to evaluate a solution. Some criteria can result from constraints that are relaxed. For instance, person-oriented consistency (continuity of care) and 


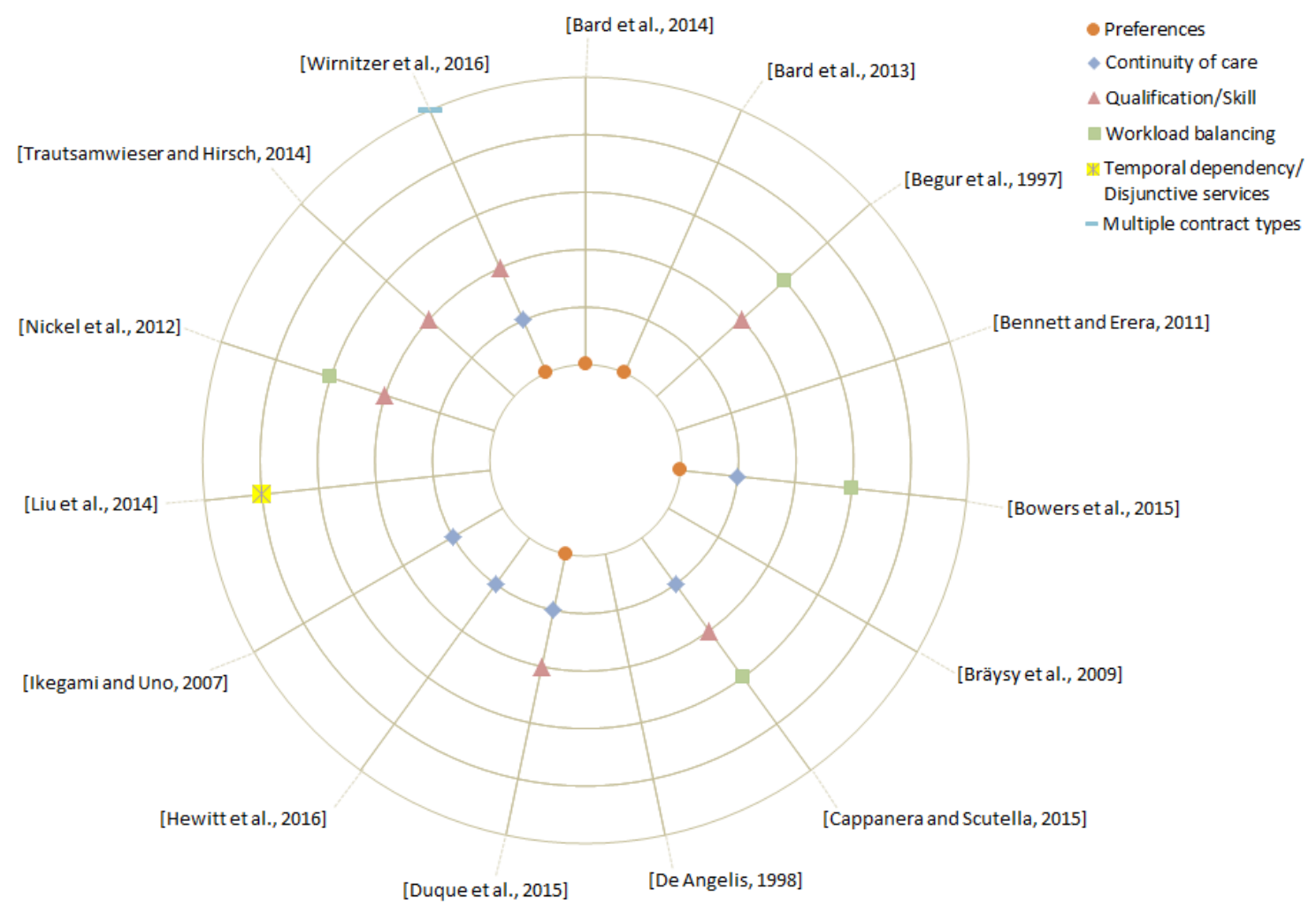

Figure 6: Publications with respect to some home care features - long planning horizon 


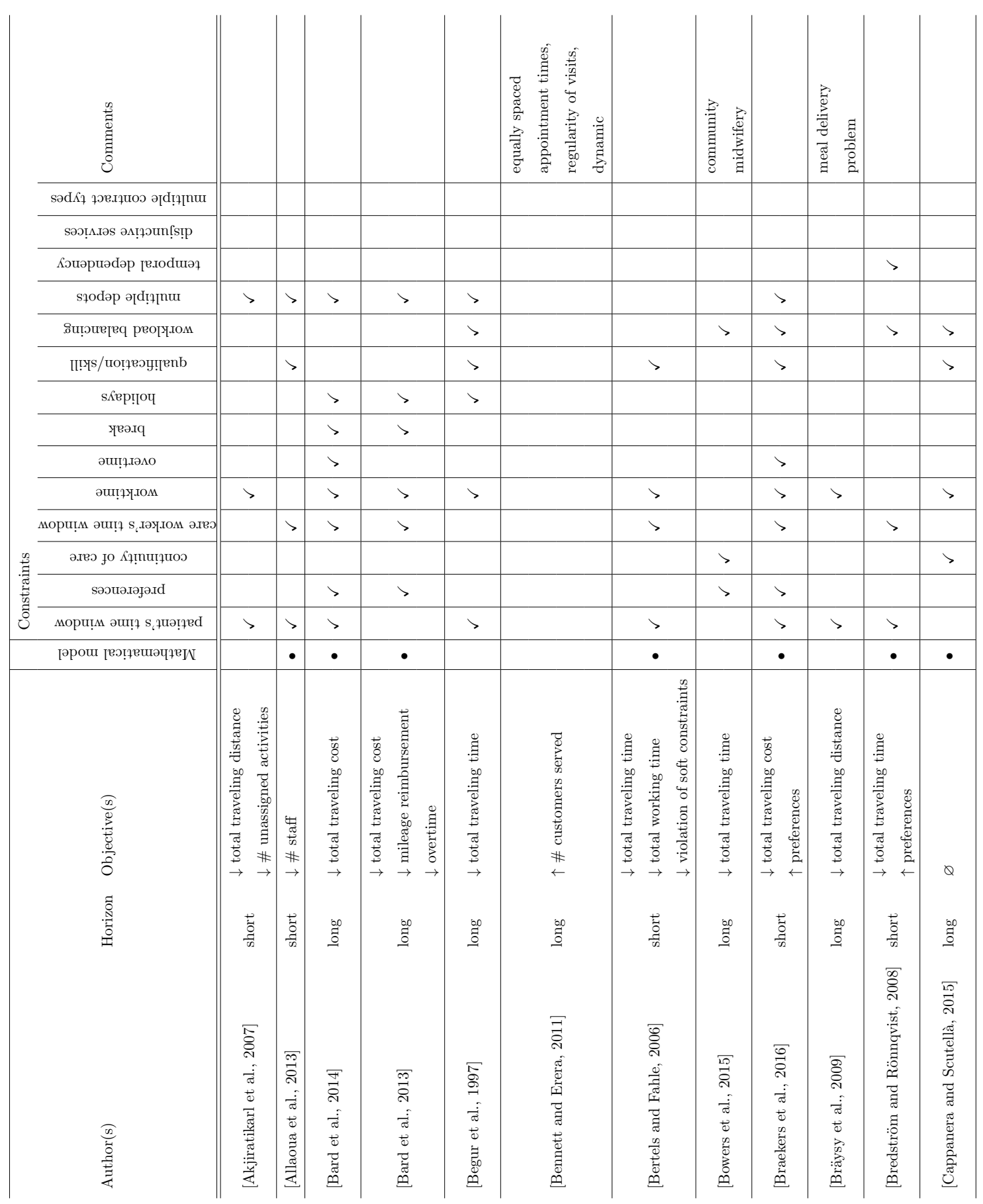

Table 2: Classification w.r.t. constraints and objectives 


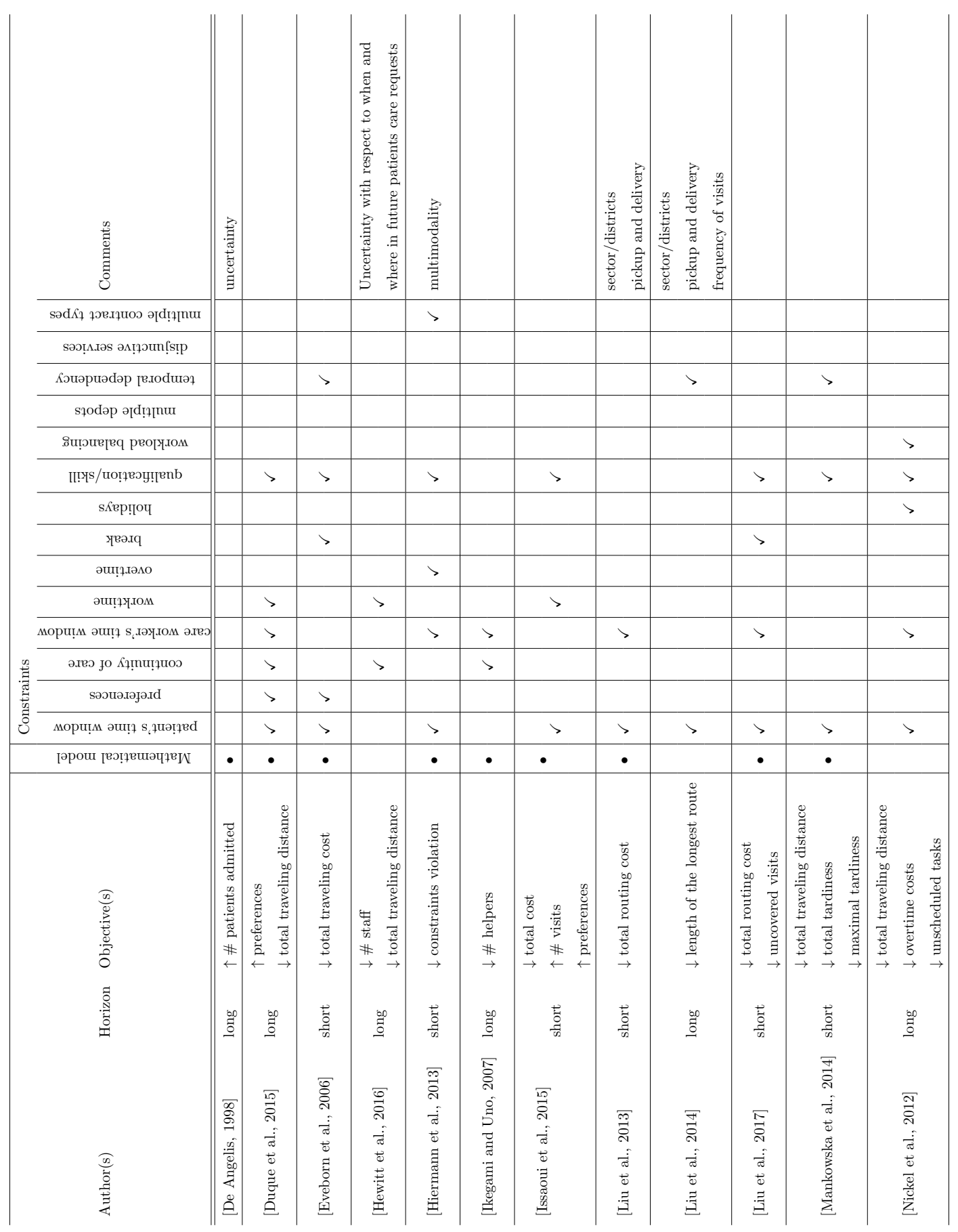

Table 3: Classification w.r.t. constraints and objectives (continued) 


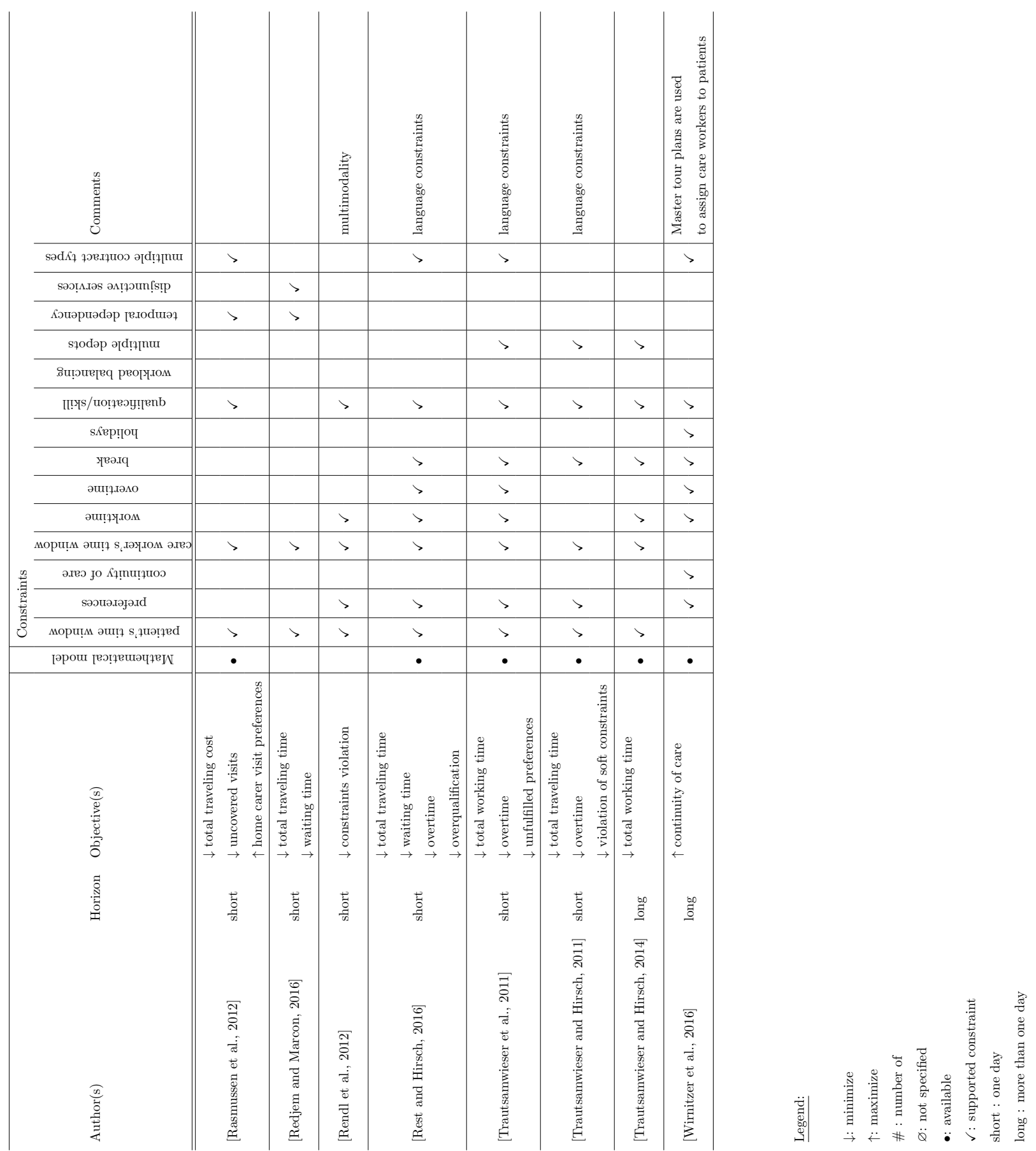

Table 4: Classification w.r.t. constraints and objectives (continued) 
workload balancing are considered as satisfaction criteria in the objective function in some papers and constraints in other works (see Section 3).

Four main types of criteria are considered in the literature: minimize total routing cost, minimize the number of unassigned services, minimize the number of care workers and maximize satisfaction. Several formulations for each type of criterion are then proposed.

\subsection{Minimize total routing cost}

The total routing cost minimization is a standard criterion in VRP. The cost covers, for example, the minimization of total traveling cost, total traveling time/duration, total travel distance and total working time, because they all are strongly correlated. In the HHCRSP literature, this criterion is the most frequently used (approximately 90\% of papers) and often corresponds to the sum of distances traveled (or the travel time incurred) by care workers. When this criterion is used in an aggregate objective function, it is generally considered the least important (with a weak weight) among other criteria such as subcontracting cost or lateness Kergosien et al., 2014, Di Gaspero and Urli, 2014, Braekers et al., 2016, Nickel et al., 2012.

\subsection{Minimize the number of unassigned services}

In some cases, the HHC provider may not have sufficient care workers to serve the entire set of patients. Services that cannot be assigned are generally subcontracted to another HHC center with a subcontracting cost Akjiratikarl et al. 2007, Hiermann et al., 2013, Kergosien et al., 2014, Nickel et al., 2012, Liu et al., 2017]. The subcontracting cost to perform a given service is always higher than the cost incurred by the provider if it is performed internally. Another formulation of the criterion is to maximize the number of assigned services or to maximize the number of cared patients Bräysy et al., 2012, Bennett, 2010, Bennett and Erera, 2011]. In both cases, the structure of the problem changes because only a subset of patients must be visited. This type of problem can be found under different names in the VRP literature: selective-TSP, maximum collection problem and the orienteering problem Vansteenwegen et al., 2011].

\subsection{Minimize the number of care workers}

In some studies, e.g., Allaoua et al., 2013, Hewitt et al., 2016, Ikegami and Uno, 2007, the number of care workers is a decision variable. Then, the objective function is to minimize the number of care workers who will serve all patients. Although this approach offers reducing staff costs, the HHC provider must be sufficiently flexible to adjust the number of employees on the routes each day.

\subsection{Maximize satisfaction}

Two types of satisfaction can be considered in the objective function: patient and care worker satisfaction. The first case refers to patient preferences, e.g., desired time windows/days/care workers. Since respecting 
all the preferences is difficult, these constraints are usually relaxed and their violation is penalized in the objective function as a disagreement of patients. For example, each violation of the time windows is penalized and each violation of non-preferential day is penalized in Kergosien et al., 2014, Mankowska et al., 2014. Braekers et al. 2016 have maximized patient preferences regarding nurses and visit times.

Wirnitzer et al. 2016 have considered six alternative objective functions related to the continuity of care, which can be considered quality measures of the satisfaction level of patients. Objective functions to minimize are: the number of different care workers per tour-cluster, the number of different care workers per patient, the relative number of different care workers per patient (relative to the patient's total visit frequency), the number of care worker-switches per patient, and the number of relative care worker-switches per patient.

In the case of care worker satisfaction, the main criterion considered in the literature is workload balancing, i.e., the decision maker must avoid a gap among workloads associated with care workers. To consider this criterion, two approaches have been proposed: balancing the number of services assigned or balancing the working time Begur et al., 1997, Yalcindag et al. 2012b. A second criterion of care worker satisfaction is the minimization of overtime, aimed at reducing the costs of an $\mathrm{HHC}$ provider and at increasing care worker satisfaction. For example, Bard et al. 2013 have considered overtime for the week (if 40 hours are reached) and Trautsamwieser et al. 2011] for the day.

Finally, Duque et al. 2015 have considered both patient and care worker satisfaction by using preference factors. Patients prefer being served in a time slot, whereas care workers prefer being scheduled to work in their desired time slot. A solution is evaluated by two objective functions. The first one is the total service level which consists in maximizing the preference factors. The second is the total distance traveled to minimize. Because the main criterion is the service level, authors proposed a hierarchical approach: maximize the first service level criterion without considering the second criterion, and then minimize the total traveled distance.

\section{Solution methods}

In this section, we present solution methods that have been proposed to solve the HHCRSP. Tables 5 and 6 list the solution methods of research published in relevant journals. They also indicate if a mathematical solver is used to solve the problem. We particularly focus only on solution methods that have been published in relevant journals that are not a simple adaptation of classic literature methods, and whose algorithms are sufficiently detailed. First, we cluster methods with respect to the considered planning horizon, i.e., single day or more than one day. Then, we distinguish exact methods from approximate methods. For the second type of methods, research is divided into three groups: single solution, population-based, and hybrid methods. 


\begin{tabular}{|c|c|c|}
\hline Author(s) & $\begin{array}{l}\text { Mathematical } \\
\text { solver }\end{array}$ & Method \\
\hline Akjiratikarl et al. 2007 & & $\begin{array}{l}\text { Particle Swarm Optimization with local improve- } \\
\text { ment procedures }\end{array}$ \\
\hline Allaoua et al. 2013 & $\checkmark$ & Matheuristic \\
\hline Bertels and Fahle, 2006. & & $\begin{array}{l}\text { Hybrid solution approach: Constraint Programming } \\
\text { with Tabu Search and Simulated Annealing }\end{array}$ \\
\hline Braekers et al. 2016 & $\checkmark$ & Large Neighborhood Search \\
\hline Bredström and Rönnqvist 2008 & $\checkmark$ & Heuristics \\
\hline Eveborn et al. 2006 & & Repeated Matching Algorithm \\
\hline Hiermann et al. 2013. & $\checkmark$ & $\begin{array}{l}\text { Variable Neighborhood Search, Simulated Anneal- } \\
\text { ing Hyper-heuristic, Memetic Algorithm and Scatter } \\
\text { Search }\end{array}$ \\
\hline Issaoui et al. 2015. & & Variable Neighborhood Descent \\
\hline Liu et al. 2013 & $\checkmark$ & $\begin{array}{l}\text { Genetic Algorithm with Local Search and Tabu } \\
\text { Search }\end{array}$ \\
\hline Liu et al. 2017 & $\checkmark$ & Branch-and-price Solution Algorithm \\
\hline Mankowska et al. 2014 & $\checkmark$ & Variable Neighborhood Search and Local Search \\
\hline Rasmussen et al. 2012 & & Branch-and-price Solution Algorithm \\
\hline Redjem and Marcon, 2016 & & Heuristic \\
\hline Rendl et al. 2012 & & $\begin{array}{l}\text { Variable Neighborhood Descent, Variable Neighbor- } \\
\text { hood Search, Simulated Annealing Hyper-heuristic, } \\
\text { Evolutionary Algorithm, and Scatter Search }\end{array}$ \\
\hline Rest and Hirsch. 2016. & & Tabu Search \\
\hline Trautsamwieser et al. 2011. & & Variable Neighborhood Search \\
\hline Trautsamwieser and Hirsch 2011. & & Variable Neighborhood Search \\
\hline
\end{tabular}

Table 5: Solution methods - short planning horizon 


\begin{tabular}{|c|c|c|c|c|}
\hline \multicolumn{3}{|l|}{ Author(s) } & \multirow{2}{*}{$\begin{array}{l}\text { Mathematical } \\
\text { solver } \\
\checkmark\end{array}$} & \multirow{2}{*}{$\begin{array}{l}\text { Method } \\
\text { Greedy Randomized Adaptive Search Procedure }\end{array}$} \\
\hline Bard et al. 2 & 2014 & & & \\
\hline Bard et al. 2 & 2013 & & \multirow[t]{5}{*}{$\checkmark$} & Heuristics based one relaxed MIP \\
\hline Begur et al. & 1997 & & & Clarke \& Wright based Heuristics \\
\hline Bennett and & \multirow{3}{*}{\multicolumn{2}{|c|}{2011}} & & Heuristics \\
\hline Bowers et al. & & & & Clarke \& Wright based Heuristic and Simulation \\
\hline Bräysy et al. & & & & $\begin{array}{l}\text { Cheapest Insertion Heuristic \& Variable Neighbour- } \\
\text { hood Descent }\end{array}$ \\
\hline \multicolumn{3}{|c|}{ Cappanera and Scutellà, 2015} & $\checkmark$ & ILP \\
\hline De Angelis 1 & 1998 & & $\checkmark$ & ILP \\
\hline Duque et al., & 2015 & & \multirow[t]{2}{*}{$\checkmark$} & Two phases using MIP and Local Search \\
\hline Hewitt et al. & 2016 & & & ConRTR algorithm \\
\hline \multicolumn{3}{|c|}{ Ikegami and Uno, 2007. } & \multirow[t]{3}{*}{$\checkmark$} & Tabu Search \\
\hline Liu et al. 20 & & & & Tabu Search combined with different Local Search \\
\hline \multicolumn{3}{|c|}{\begin{tabular}{|l|l|} 
Nickel et al. & 2012 \\
\end{tabular}} & & $\begin{array}{l}\text { Heuistics using Constraint Programming, Tabu } \\
\text { Search and Adaptive Large Neighborhood Search }\end{array}$ \\
\hline \multicolumn{3}{|c|}{ Trautsamwieser and Hirsch 2014} & & $\begin{array}{l}\text { Branch-and-price Solution Algorithm and Variable } \\
\text { Neighborhood Search }\end{array}$ \\
\hline Wirnitzer et & al. 2016 & & $\checkmark$ & ILP \\
\hline
\end{tabular}

Table 6: Solution methods - long planning horizon 
Due to the lack of standard benchmarking, most approaches have not been extensively compared. Thus, it might not always be possible to ascertain the effectiveness of a method with respect to others. However, solution methods can be identified according to the "winning" strategies proposed by Vidal et al. 2013b or according to the size of the solved instances. Table 7 lists "winning" strategies for each study in the literature; these are described later. This table identifies the features of each method (from each work) and provides an overview of OR techniques used to design efficient methods to solve the HHCRSP. Each line refers to a paper that includes one or several methods. The first two columns refer to the search space (SP). Among columns, RELAXATION indicates if the exploration of infeasible solutions is allowed, and most of studies consider only the exploration of feasible solutions. IND.REPRESENT. specifies the use of indirect representations (indirect encoding) of solutions and is only considered by five studies. The next five columns are related to the neighborhoods (NEIGHBOUR.). The first, MULT. NEIGHB., represents the use of different types of neighborhoods, and the next one, ENUMERATIVE, indicates whether the neighborhood solutions are enumerated in a polynomial-time. Although both features are present in most of works, the consecutive three features that specify the use of pruning procedures (PRUNING), use of large neighborhoods (LARGE) and use of solution recombination (RECOMBIN.), appear less frequently. The successive four columns are related to the search trajectory (TRAJEC.), which represents the path of solutions explored by the method through the neighborhood search space. The search trajectory can include random steps (RANDOMNESS), which is true for most papers, and can be continuous (CONTINUOUS), discontinuous (DISCONTINUOUS) or mixed (MIXED). In the continuous case, few changes can be seen between two successively explored solutions, whereas in the discontinuous case, both successively explored solutions can be very different. Within the considered literature, most of the works include a continuous trajectory. The next four columns, memories and control (MEM \& CONTROL), are related to how the past search history is managed: using a set of solutions (POPULATION), diversity steps to explore a new search space (DIV. MANAG.), parameter adaption (PARAM. ADAPT.) (e.g., parameter to limit the frequency of use of operator, or parameter to measure the quality of solution), and advanced guidance mechanisms (GUIDANCE) to manage the intensification and diversification of the search. Although these MEM \& CONTROL techniques are not extensively considered and they are not simple to design/implement, it might be interesting to integrate them to make solution methods more efficient. Finally, the last columns indicate whether a hybrid algorithm technique is used (HYBRIDIZATION), whether parts of the problem are solved by using integer programming (MATHEURISTICS), whether any parallelism techniques are implemented (PARALLELISM), and whether the problem is decomposed into several sub-problems and solved separately (DECOMPOSITION).

After presenting the overview of "winning" strategies and also identifying these strategies for each study considered (as shown in Table 7), in the following parts, we present the most interesting solution methods 


\begin{tabular}{|c|c|c|c|c|c|c|c|c|c|c|c|c|c|c|c|c|c|c|c|c|c|c|c|}
\hline & NOILISOdNO৩Ađ & & $>$ & & & & $>$ & & & $>$ & $>$ & & & & $>$ & & & & $>$ & & & & \\
\hline & ISITHTTVYVd & & & & & & $>$ & & & & & & & & & & & & & & & & \\
\hline & SDILSIYกAHLVIN & & $>$ & & & $>$ & & & & $>$ & $>$ & $>$ & & $>$ & & & & & $>$ & $>$ & & & \\
\hline & NOILVZIđIчgגн & & $>$ & & & $>$ & & & & $>$ & $>$ & & & $>$ & & & $>$ & & $>$ & $>$ & & & \\
\hline 㞱 & НОNVСIกり & $>$ & & & & $>$ & & & & & & & & & & & $>$ & & $>$ & & $>$ & & \\
\hline $\int^{2}$ & 'LdVCV 'NVUVd & & & $>$ & & & & & & & & & & & & & $>$ & & $>$ & & $>$ & & $>$ \\
\hline$\dot{\Sigma}$ & 'DVNVI $\Lambda$ IA & & & & & $>$ & & $>$ & & & & & $>$ & & & & & & & & $>$ & & \\
\hline $\bar{\Sigma}$ & NOILVTAdOd & $>$ & & & & $>$ & & & & & & & & $>$ & & $>$ & & & & $>$ & & & \\
\hline & वнХХIN & & & & & & & $>$ & & & & & & $>$ & & & & & & $>$ & & & \\
\hline 兽 & SnONILNODSIC & & & & & & & & & & & & & $>$ & & & & & & $>$ & & $>$ & \\
\hline 突 & SחOANILNOP & $>$ & & $>$ & & & & & $>$ & & $>$ & $>$ & $>$ & $>$ & & $>$ & $>$ & $>$ & $>$ & $>$ & $>$ & & $>$ \\
\hline & SSANNOCNVY & $>$ & & $>$ & & $>$ & & $>$ & & $>$ & $>$ & $>$ & & $>$ & & $>$ & $>$ & & $>$ & $>$ & & & $>$ \\
\hline & ·NIGNOगЯч & & & & & & & & $>$ & & & & & $>$ & & & & & & $>$ & & & \\
\hline 它 & ヨりบVT & & & & & & & $>$ & & & & & & $>$ & & & & & $>$ & $>$ & & & \\
\hline$\underset{T}{\oplus}$ & DNINAYd & & & & & $>$ & & & & & & & & $>$ & & & & & & $>$ & & $>$ & \\
\hline 弐 & 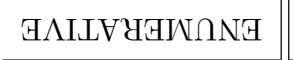 & $>$ & & $>$ & & & & & & & $>$ & $>$ & $>$ & $>$ & & $>$ & $>$ & $>$ & & $>$ & & $>$ & \\
\hline & 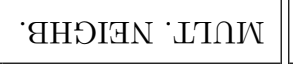 & $>$ & & $>$ & & $>$ & & $>$ & $>$ & & $>$ & $>$ & $>$ & $>$ & $>$ & $>$ & $>$ & $>$ & $>$ & $>$ & & $>$ & $>$ \\
\hline & 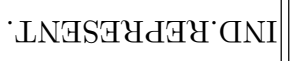 & $>$ & & & & & & & & & & & & $>$ & & $>$ & & $>$ & & $>$ & $>$ & & \\
\hline & NOILVXVTAY & $>$ & & & & & & & & & & & & & & $>$ & $>$ & & & & $>$ & & $>$ \\
\hline & & 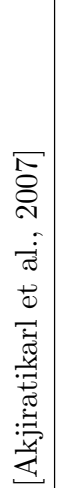 & 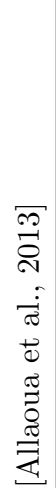 & 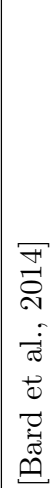 & 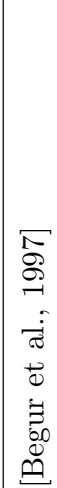 & 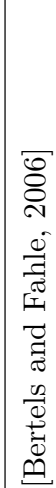 & 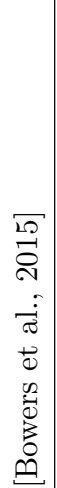 & 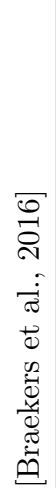 & 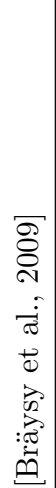 & 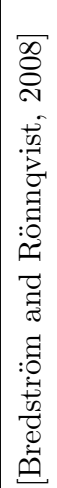 & 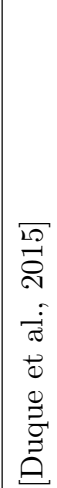 & 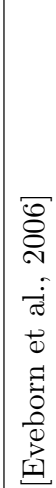 & 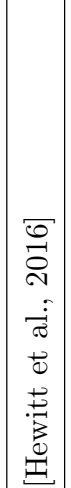 & 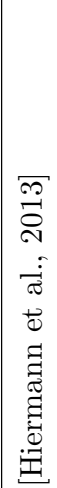 & 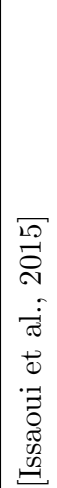 & 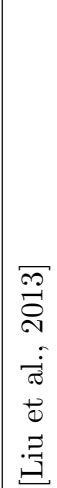 & 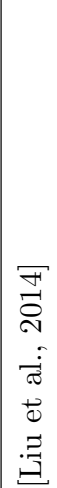 & 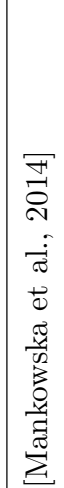 & 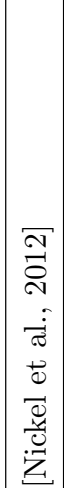 & 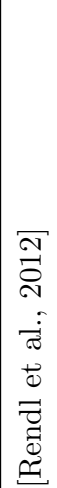 & 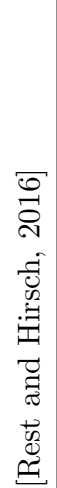 & 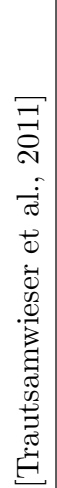 & 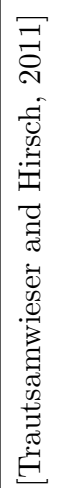 \\
\hline
\end{tabular}

Table 7: Metaheuristics for the HHCRSP and winning strategies (Vidal et al. 2013a]) used 
according to the previously stated structure, i.e., considered planning horizon and type of method.

\subsection{Methods handling a short planning horizon}

In this section, we focus only on methods designed for a one-day planning horizon.

\subsubsection{Exact methods}

Rasmussen et al. 2012 have designed a dynamic column generation approach embedded into a branchand-price framework for the HHCRSP in which temporal dependencies between the start times of visits have to be respected. The studied problem is similar to that presented in Bredstrom and Ronnqvist, 2007]. A branching scheme is introduced to handle both integrality and precedence constraints, which are relaxed in the master problem. The master problem is used to determine a minimum cost schedule for each care worker among the feasible schedules. These schedules are generated by a pricing problem which is an elementary shortest path problem with time windows. The pricing problem is composed of as many independent problems as the number of care workers, because each care worker has different features. This approach was tested in real instances (7-15 care workers, 60-150 services), randomly generated instances (715 care workers, 150 services), and instances from the literature Bredstrom and Ronnqvist, 2007] (4-16 care workers, 20-80 services). Computational results show that the proposed solution approach outperforms the algorithm of [Bredstrom and Ronnqvist, 2007], which is a similar method using a set partitioning formulation and deriving properties. The method of [Rasmussen et al., 2012] can improve the best-known solutions and/or compute optimal solutions faster in some cases.

Liu et al., 2017 have also proposed a branch-and-price algorithm for solving an HHCRSP in which a lunch break has to be scheduled on each route of the care workers and some patients cannot be visited. The problem is decomposed into a master problem using a set-partitioning formulation and pricing subproblems that are solved by a label-correcting algorithm adapted to take the lunch breaks into account. Several improvement techniques have been proposed: extension rules, dominance rules, cutting-edge column generation strategies, and hierarchical branching scheme. Experimental results shows the effectiveness of the proposed algorithm comparing to the results obtained by solving the mathematical model using CPLEX solver. Two types of instances have been used: instances derived from the literature (Solomon's VRPPTW benchmarks) and real-life data provided by an HHC company in China.

\subsubsection{Approximate methods}

Single solution methods. Redjem and Marcon, 2016 proposed a two-step heuristic, the Care-givers routing heuristic $(\mathrm{CRH})$ to solve a routing problem in which each service has been pre-assigned to a care worker at a previous tactical level of decision. The first step is dedicated to determining the shortest route for each care worker without taking into account non-overlapping and precedence constraints between services. This 
step is performed by enumerating all possible routes. The second step aims to satisfy non-overlapping and precedence constraints using shifting or swapping moves. The proposed heuristic was tested on three sets of instances inspired from real data composed of up to 60 patients, 30 care workers, and 240 services.

Eveborn et al. 2006] presented a decision support system, Laps Care, for care workers planning HHC structures in Sweden. Each patient visit has a time window and requires a set of skills. Breaks have to be scheduled in the routes of care givers and the objective function is to minimize the costs of the travel time, preferences, and/or inconvenient working hours. They have used a set-partitioning model to provide a flexible architecture in which patients are divided into subgroups and assigned to the routes of care workers. The problem is solved by using a repeated matching algorithm that starts from an initial matching and iteratively creates a new matching by local improvements and splitting. This approach enables attaching priorities to different aspects of the solution, such as travel times and patients' preferred care workers. Numerical tests on real instances of 12-20 care workers and 86-123 services show savings of $7 \%$ of the total working time and $20 \%$ of the travel time compared with manual planning solutions, with computational times of less than three minutes.

An adaptive variable neighborhood search (AVNS) algorithm was proposed by Mankowska et al., 2014 to solve daily routing and scheduling problems. Patients need services that are only characterized by a soft time window and a qualification. Synchronization and minimum/maximum time lags between services are considered. In this work, solutions are encoded with the use of a matrix that consists of the assignments of patients to care worker routes and the synchronization information. The initial solution is obtained by a greedy heuristic in which urgent patients are scheduled first and all patients are assigned to care givers who can arrive at the considered patient's home earliest among all of the other suitably qualified care workers. In the next step, eight different neighborhood structures are defined to improve this initial solution. These eight neighborhoods are grouped using the following four moves: shift a patient within a route (without changing the care worker), swap two patients inside a route, shift a patient from a route to another (changing the assigned care worker), and swap two patients belonging to two different routes. Then, these neighborhoods are sorted by decreasing performance to start with the one that has the highest potential for obtaining good solutions. This algorithm is tested on randomly generated instances having 10 to 300 patients and 3 to 40 care workers, and solutions are compared with the CPLEX solver solution. In ten hours, CPLEX implementation is able to find exact solutions for very small instances, composed of 10 patients and 3 care workers and feasible solutions for instances of 25 patients and 5 care workers, with an average gap of $35.1 \%$ from the lower bound. For larger instances, CPLEX does not find any feasible solutions, except in one case. Conversely, the heuristic is able to find optimal solutions for very small instances in less than one second and find better solutions than CPLEX for instances of 25 patients. This heuristic finds solutions in less than 6 seconds for instances up to 100 patients and 20 care workers, but it needs more than 2 hours if the number 
of patients reaches 300 .

Trautsamwieser et al. 2011 studied a daily planning problem of HHC services. The problem is laden with assignment constraints (qualification, language, rejection), working time restrictions, time windows, and mandatory break times. The objective function is to minimize the sum of driving and waiting times, and the dissatisfaction levels of patients and care givers. Authors have proposed a VNS algorithm for the HHCRSP in which the initial solution is obtained with a simple heuristic that assigns services to care workers based on the mean of their time windows while ensuring qualification and working-time constraints. This heuristic favors the closest feasible route assignment based on the time window of a service or working time of a care worker. Then, a shaking procedure and a local search procedure are combined. The shaking procedure is used to diversify the search by providing guidance toward new parts of the solution space via relocations of segments of patients from one tour to another and cross exchanging of two segments of different tours. Then, routes are improved using a 3-opt neighborhood. Computational experiments are run over instances on both randomly generated instances with 4 to 20 care workers and 20 to 100 services, and real instances with 13 to 75 care workers, 86 to 411 patients and 86 to 512 services. The results on randomly generated instances are compared with those obtained by Xpress solver, and gaps less than $2 \%$ are observed. Conversely, results of the real problem instances are compared with an actual plan, and improvement of up to $50 \%$ is observed. Although all of these results are promising, the proposed algorithm does require long computational times.

Liu et al. 2013 addressed the HHCRSP with mixed backhauls in which drugs and medical devices must be delivered from a pharmacy to patient homes and biological samples must be picked up from patient homes. The problem is similar to a pickup and delivery problem with precedence constraints according to the type of demands (delivery from depot to patient, delivery from a hospital to patient, pickup from a patient to depot and pickup from a patient to a medical lab). Two solution methods are proposed: a hybrid genetic algorithm (GA) and a Tabu Search (TS) method. Only the TS approach is discussed in this part; the GA will be described in the section dedicated to population-based methods. The proposed TS method starts with the best feasible solution of the initial population of the proposed GA. Neighborhoods are defined by two local moves; the first moves a node (a patient) from one route to another, and the second exchanges two nodes of different routes. After each move, the modified routes are re-optimized by a constructive method followed by a local search. If there is no improvement within a specified number of iterations, the TS is restarted with the second-best initial population solution of the GA. The TS algorithm is evaluated on two sets of instances based on VRPTW-based instances, and on VRP with mixed backhauls and time window (VRPMBTW) benchmarks with two stopping criteria: (i) maximum number of iterations and maximum number of iterations without improvement (TS1), and (ii) the same computation time as GA (TS2). In the first set, instances are generated by modifying the examples of Solomon, 1987] and Gehring and Homberger. 1999]. The Solomon, 1987's examples are composed of 40 to 120 services and 10 to 20 care workers, whereas 
those of Gehring and Homberger, 1999 use 200 services and 50 care workers. For this first set of instances, an average cost saving of $17.1 \%$ is observed between CPLEX solutions and the best solutions of TS1 and TS2. Computational times remain acceptable with on average 863.5 seconds for TS1 and 951.8 seconds for TS2. Conversely, the second set regroups Hasama et al. 1998 instances that are composed of 100 patients and up to 22 care workers, in which certain number of patients, i.e., 10\%, 30\% or $50 \%$ of 100 patients, are considered backhaul patients. For these instances, only TS2 is tested; it is compared with the SA heuristic defined in Hasama et al. 1998 and provides $6.27 \%$ of cost reductions on average.

Rest and Hirsch, 2016] studied a daily home care routing and scheduling problem (with qualification levels, hard time windows for patients, maximum working time and breaks for care workers) that considers time-dependent travel times out of the timetables from public transport service providers. The authors proposed a Tabu Search algorithm based on the unified approach of Cordeau et al. 2001. The initial solution is computed by a best insertion heuristic. First, jobs that represent patient visits are sorted according to the center of time windows. Then, based on this order, jobs are inserted in the routes by trying to satisfy qualification and preference requirements. Some jobs may not be inserted in the solution and lead to unfeasible solutions. At the end, all unassigned jobs are inserted even if it implies some constraint violation. These violations are quantified and taken into account in the objective function in order to guide the search toward feasible solutions. In such cases, they are postponed and added later. The used neighborhood consists in relocating a job, i.e. a job is removed from a route and is inserted in another route. The TS proposed by this study is declined in three versions regarding the size of the neighborhood. The version named TS explores the entire neighborhood. The second one named TSAS explores the neighborhood of fixed size. The last version named TSDYN, dynamically adapts the size of the neighborhood. Numerical experiments have been conducted on real instances with 106 to 202 jobs and 16 to 45 care workers. The mathematical solver FICO Xpress 7.0 is not able to solve all these real instances and only solves to optimality small instances up to 6 care workers and 30 jobs. With a time limit of 600 seconds, three versions of TS are compared with each other by applying them to the real data set. However, it is difficult to conclude that one version outperforms the others due to the fact that their performances change according to the instance.

Bräysy et al. 2009 studied a home meal delivery problem. The problem is modeled as a multiple traveling salesman problem with time windows and solved using a commercial solution: SPIDER Designer 4.0. The kernel of this solver is based on a variable neighborhood descent (VND) metaheuristic. An extended version of the cheapest insertion heuristic computes the initial solution. The solution is then improved with successive intensification and diversification phases. The diversification phase is an iterative "destroy and reinsert" method Pisinger and Ropke, 2007b. For the VND intensification phase, a set of well known local search operators in routing literature are used: Relocate, 2-opt*, CROSS-exchange, 2-ot, 3-opt and Tour Depletion. Experimental results are presented from real data sets from the Finnish city of Jyväskylä. Seven 
scenarios are introduced to compare the author's approach with a manual planning approach. The proposed method allows to improve the total distance traveled between $12.41 \%$ and $51.24 \%$.

Population-based methods. To tackle real-world HHCRSP where each care worker is constrained by a maximum working time and patient visits are characterized by time windows, Akjiratikarl et al. 2007 have introduced a particle swarm optimization (PSO) based algorithm. A particle, which models a solution in the search space, is encoded through a matrix (the size is the number of care workers and number of services) to determine assignment decisions between services and care workers and to build the visiting sequence of services for each care worker. Then, initial solutions are generated based on earliest start time priority and minimum distance considerations. The goal of the proposed algorithm is to explore the solution space globally and use local improvement procedures to fine-tune search around local areas. Computational experiments are conducted on different versions of this particle swarm optimization algorithm in which some parts of the algorithm are removed. Computational experiments are run on real world instances consisting of over 100 services, 50 patients and 12 care workers per day. Solutions of the proposed PSO algorithm are compared with those obtained from manual planning and from ILOG Dispatcher 4.0. The complete version of the algorithm outperforms the other versions and the manual planning case. Moreover, the PSO algorithm is able to provide up to $31 \%$ of total mileage savings within 3.5 minutes when compared to the ILOG Dispatcher.

As mentioned in the previous section, Liu et al. 2013 have also developed a GA for the HHCRSP. This algorithm is inspired by the work of Prins, 2004, in which the solution is encoded by chromosome based on patient permutation. The initial population of the proposed GA is generated by three saving-based and one nearest-neighbor heuristic. Crossover can generate non-feasible solutions; therefore, it is repeated until at least one offspring corresponds to a feasible solution. This GA is tested in the same setting of the previously explained TS method used by Liu et al. 2013. The results indicate that this algorithm provides up to $16.4 \%$ cost savings with respect to the solutions of the CPLEX solver and $6.35 \%$ savings compared to Hasama et al. 1998's SA. These computational tests do not clearly allow for the establish of dominance relationship between GA and the two versions of TS.

Hybrid methods. Bredström and Rönnqvist, 2008 developed a hybrid approach by employing heuristics for the generation of columns within a column generation framework to solve the HHCRSP with time windows and additional temporal constraints. The proposed approach is summarized in seven steps, which are divided into two stages. The first stage consists of three steps and produces an initial feasible solution. First, each service is pre-assigned to a subset of care workers; then, a relaxed mixed-integer program based on these pre-assignments is solved to refine them and possibly identify services that cannot be assigned to a care worker. Finally, based on these assignments, a restricted mixed-integer program is solved to find a feasible solution. Conversely, the second stage includes four steps that are repeated. The goal is to improve the 
best feasible solution by randomly generating new pre-assignments based on this solution and solving the resulting restricted mixed-integer program within a predetermined time limit. The proposed approach is tested on randomly generated instances that include up to 16 care workers and 80 services; results are compared with those of the CPLEX solver. An interesting sensitivity analysis is also performed to evaluate the effect of synchronization constraints, time window sizes and percentage of synchronized visits. According to the results, the proposed heuristic is efficient and in 2 minutes finds solutions as good as those computed by the CPLEX solver in 60 minutes.

Bertels and Fahle, 2006 have studied the HHCRSP where each patient's visit is characterized by a soft and a hard time window, the qualifications required, and care worker preferences. The authors propose a hybrid algorithm based on constraint programming (CP), linear programming (LP) and tabu search (TS) or simulated annealing (SA). The proposed algorithm consists of a two-phase approach: An assignment of services to care workers is performed in the first phase, and visiting sequences for each care worker are generated in the second phase. The algorithm first starts with a pre-processing step to enumerate possible assignments according to skill and time window constraints. Then, CP is used to associate each care worker with a list of services that are then sequenced with the help of LP. In the next step, either SA or TS is applied to improve this solution until a total run limit of 900 seconds is reached. Numerical experiments are conducted on 120 artificial test instances including up to 50 care workers, 200 patients and 600 services. Results show that the CP and TS combination represents the best solution method by providing an improvement in all 12 instances when compared with standalone CP, TS or SA solutions. Conversely, the combination of CP and $\mathrm{SA}$ is also able to improve the solutions of 8 instances (instead of 12), but these improvements are weaker than those obtained by the combination of CP and TS.

A decomposition approach is proposed in Allaoua et al. 2013 where they have clustered services in the first stage that share the same qualification requirements. Then, a TSPTW problem is solved in the second stage for each cluster. Unlike the previous method, a mixed integer linear programming approach is used instead of CP. The proposed approach is tested on 90 randomly generated instances having up to 9 care workers and 30 services and solutions are compared with respect to the CPLEX solver. In 50.52\% of instances, the solution found by this approach has the same objective value as the one found by CPLEX with a limited running time.

Rendl et al. 2012 and Hiermann et al. 2013 developed solution methods for multimodal in the HHCRSP. To represent a solution, they used a natural encoding scheme that associates each care worker with a list of services in which each service is only considered within a single list. They have studied four metaheuristic approaches: VNS, memetic algorithm (MA), SA, and scatter search (SS). The initial solutions of these metaheuristics are randomly computed or generated by using CP. All alternative cases are evaluated based on realistic instances composed of $482 / 518$ care workers and $679 / 717$ services. It is observed that all 
approaches provide solutions having a low number of care workers, i.e., approximately $35 \%$ of the available care workers are used. Computational results show that all solutions provide satisfactory results in reasonable times, and some of them strongly benefit from valid initial solutions from a CP-based approach. Among all metaheuristic approaches, the MA outperforms the others independently of the solution used initially. Results have been enforced by the recent success of MA in solving multi-attribute vehicle routing problems in Vidal et al. 2013a.

Braekers et al., 2016 modeled a daily home care routing and scheduling problem as a bi-objective problem. The first objective is to minimize the total costs (sum of travel costs and overtime costs) and the second objective is to minimize patient inconvenience regarding care workers and visit times. Authors have proposed a MILP formulation with some enhancements (branching priority on new additional variables and adding some valid inequalities). They have also proposed dynamic programming to solve the bi-objective problem with a single route. Finally, they have developed a metaheuristic to find the set of Pareto optimal or efficient solutions. This is based on the multi-directional local search framework proposed by [Tricoire, 2012. The main idea of the multi-directional local search is to select a solution among a set of non-dominated solutions, improve the selected solution using a large neighborhood search for each objective (a solution is obtained for each objective) and update the set of non-dominated solutions according to the resulting solutions. The large neighborhood search uses 12 standard removal operators (6 for each objective) and 4 standard insertion operators from the literature, most of which were introduced in Pisinger and Ropke, 2007a. This solution approach is based on 90 instances composed of 30 small test instances up to 25 services and 60 real size instances with up to 300 services. Finally, the solution approach is compared with an exact approach on small instances that shows the efficiency of the approach.

\subsection{Methods of handling a long (multiple days) planning horizon}

\subsubsection{Exact methods}

Trautsamwieser and Hirsch, 2014 have designed a branch-price-and-cut (BPC) algorithm to solve a weekly HHCRSP in which patients can be visited one or several times during the week by appropriately skilled care workers and during predefined time windows. The working times of care workers are constrained by maximum working time per day or per week, and by breaks. The problem is decomposed based on a Dantzig-Wolfe decomposition approach where the master problem combines daily feasible care worker routes to provide a weekly schedule while minimizing the total working time. These daily feasible routes are obtained by solving the pricing problems of the decomposition. An important contribution of this work is based on a set of branching rules applied to obtain integer solutions. In this framework, the initial solution is obtained with the use of a VNS algorithm used to provide good upper bounds to the BPC algorithm. The proposed approach is applied to solve real-life based instances including up to 9 care workers, 45 patients and 203 services within one hour. Cappanera and Scutellà, 2015 developed an integer linear programming (ILP) formulation for the 
weekly planning of the HHCRSP where patterns from different generation policies are used to visit patients according to the continuity of care and skill compatibility issues. In particular, maximum daily working times of care workers are considered while taking into account the workload balancing objective. Firstly, the proposed model is tested on realistic instances with the use of CPLEX solver where these instances are composed of 40 to 162 patients and 4 to 11 care workers. Then, data sets developed by Nickel et al. 2012] and Jensen, 2012 are used to compare the quality of the proposed model's solutions with ones from literature. Wirnitzer et al. 2016 proposed a mixed integer programming model for the monthly planning of the HHCRSP where weekly master tour plans are predefined and the care workers should be assigned to tours while taking into account continuity of care, availability, compatibility, and capacity restrictions. The proposed model is tested on more than 45 instances, including a real case which is composed of up to 138 master tours, 37 care workers, 143 patients and 1114 visits in a week. All the cases are solved via Gurobi solver. The results show that using this approach improves continuity of care compared to the manual solution.

\subsubsection{Approximate methods}

Single solution methods. Bard et al. 2014 and Bard et al., 2013 developed, respectively, a sequential and a parallel greedy randomized adaptive search procedure (GRASP) to solve the HHCRSP. In this problem, they considered two types of care workers, called physical therapists and physical therapist assistants and some patients who have to consult at least once with a physical therapist. Experimental results tend to show that the sequential GRASP is more efficient. In Bard et al. 2014, the initial solution is built in three steps. In the first step, a day is chosen for each patient who has to meet a physical therapist. In the second step, a pair (care worker, day) is randomly selected. Priority is given to days that maximize global care workers' availability followed by care workers with the lowest wage rates. This pair is called a route. In the third step, patients are randomly selected to complete the route. Steps 2 and 3 are repeated until all routes are completed. Finally, this initial solution is improved by intensive local search procedures using inter and intra route insertions and swaps. The algorithm is tested on both real (5 instances) and randomly (80 instances) generated instances. Real instances involve 38 to 113 patients, 93 to 278 visits and 4 to 12 care workers for a planning horizon of one week. The results of the real instances show that $26.57 \%$ of cost reduction is obtained with respect to the actual planning. When the size of instances grows, an average cost reduction of $5.67 \%$ for four of the real instances is obtained. The sequential GRASP outperforms the parallel GRASP implemented in Bard et al. 2013. Similar observations are made on randomly generated instances.

Duque et al. 2015 have proposed a two-stage algorithm for the HHCRSP, where two objectives are considered: Service level maximization is considered in the first stage and total distance minimization is considered in the second. The first stage exhaustively enumerates patterns of days in which patient services can be provided. Based on these patterns, a limited number of feasible schemes are generated in order to 
choose the best assignment between care workers and patients. Starting from the first stage solution, the second stage minimizes the total distance while accepting the deterioration of the solution with a certain tolerance. To this end, a randomized local search algorithm that swaps patients from different visits is implemented. The proposed algorithm is tested on 30 random instances that consist of 26 to 109 patients and 3 to 25 care workers. The algorithm provides solutions with a good service level, over $89.35 \%$ of the theoretical value. Moreover, on average, a reduction up to $11.67 \%$ in the total distance is also observed.

Bowers et al. 2015 have presented a heuristic based on Clarke and Wright's algorithm (cf. Clarke and Wright, 1964) to handle an HHCRSP in a community midwifery circumstance. The studied problem corresponds to a multiple traveling salesman problem where continuity of care, patient preference satisfaction, and travel time reduction must be taken into account. Clarke and Wright's method is only based on traveling cost. In order to take into account the patients's preference, the methods are revised and implemented. The method is embedded in a discrete-event simulation to validate the proposed approach. Finally, five simulation experiments with different problem settings are conducted and suggest that continuity of care can be achieved with a small increase in the total traveling time.

Hewitt et al. 2016 have compared several planning strategies to solve a variant of the consistent vehicle routing problem (ConVRP) using the ConRTR algorithm proposed by Groër et al. 2009. Authors have also considered a stochastic setting where there is uncertainty with respect to when and where future patients will request care. To do so, an adaptation of the ConRTR is made based on a point estimate of future patient requests. The ConRTR algorithm consists in creating template routes from which the route for each day will be defined. The template routes are created using the Clarke and Wright algorithm and is improved using a local search with three types of moves (one-point, two-point and two-opt move) and a diversification phase. Three planning strategies are compared: week-by-week, long-term, and long-term planning under uncertainty planning approaches. It is observed that savings in travel time and nurse-staffing requirements can be realized by planning for a period of time that is much longer than what is typically seen in the vehicle routing literature.

Hybrid methods. Ikegami and Uno, 2007 have focused on determining the number of care workers required while taking into account continuity of care, maximum number of services per care worker and time window constraints. They have proposed an upper bound and two lower bound calculation approaches to define a range for the number of care workers. Relaxations based on mathematical model formulation is used for the lower bounds whereas a TS approach is adopted for the upper bound. Real data sets with up to 31 services and 18 care workers per day are used to test the proposed methodology.

Liu et al. 2014 have studied an extension of a classical periodic vehicle routing Problem with Time Windows where three types of demands are considered: transportation of drugs/medical devices, delivery of special drugs, and delivery of blood samples. Each patient is defined by a number of visits within a planning 
horizon and has a set of possible combinations of visit days. A combination of TS and local search procedures is developed to solve the problem. The proposed TS algorithm is based on the general TS framework of Cordeau et al., 1997. The authors have considered an augmented cost function to take into account strategic oscillation between feasible and infeasible spaces. The initial solution is generated using an insertion heuristic while guaranteeing only precedence constraints. Thus, time window constraints cannot be respected and lead to an infeasible solution. This step is then followed by a neighborhood exploration operation by inter-routes movements. In this paper, the TS algorithm is hybridized by intra-route local search procedures to be able to intensify the search. To this end, 1-1 exchange, 1-0 exchange and 2-Opt exchange algorithms are implemented by allowing both feasible and infeasible moves. Large computational experiments are conducted on different test instances, including extensions of some instances in the literature ([Solomon, 1987] and Gehring and Homberger, 1999]), as well as real-life cases. Results show that it is profitable to consider infeasible solutions during the research procedure. In particular, the use of the TS algorithm on 5 real instances with 2 care workers, 49-58 patients and 5 days leads to an improvement from $9 \%$ to $16 \%$ relative to the implemented solutions.

Nickel et al. 2012 have proposed a two-stage solution approach combining constraint programming based heuristic and adaptative large neighborhood search to solve an HHCRSP. Patients are characterized by a time window, a qualification required, a frequency of visit, and a set of possible shift combinations of visits. In the first stage, the $\mathrm{CP}$ based heuristic computes an initial feasible solution in which unscheduled services can exist. In that case, these unscheduled services are assigned to a dummy nurse. In the second stage, an adaptative large neighborhood search improves the initial feasible solution. For that purpose, for each iteration, a given number of services are removed from the current solution and reinserted. In this way, new spaces are explored. The two-stage solution approach is implemented to solve real size instances on 7 days, with 11 and 12 nurses, 287 and 361 services.

In summary, a large number of methods have been proposed to solve the HHCRSP. Figure 7 summarizes the types of approaches developed. Most of them are single solution methods or hybrid algorithms. VNS is the most used after the simple dedicated heuristic methods. This method seems to be appropriate to solve the HHCRSP because it is simple to implement and combines an intensification phase with a diversification phase during the exploration of solution space. Among all heuristics used to build initial solutions, we can find two types of approaches according to how the assignment and routing decisions are solved, simultaneously or in two successive phases. In the second case, care workers are first assigned to patients on a geographical basis to match the skills required by a service at the patient's home with the care worker's skill; second, the schedule of each care worker is determined. Finally, we can also note that most of the proposed methods use a direct encoding solution, and few parallel algorithms have been proposed. 


\begin{tabular}{|c|c|c|c|c|c|}
\hline Author(s) & $\begin{array}{l}\text { Instance } \\
\text { Type }\end{array}$ & $\begin{array}{l}\text { Planning } \\
\text { Horizon }\end{array}$ & $\begin{array}{l}\text { Max. Num. of } \\
\text { Care Workers }\end{array}$ & $\begin{array}{l}\text { Max. Num. of } \\
\text { Patients }\end{array}$ & $\begin{array}{l}\text { Max. Num. of } \\
\text { Services }\end{array}$ \\
\hline Akjiratikarl et al. 2007 & $\mathrm{R}$ & Short & 12 & 100 & - \\
\hline Allaoua et al. 2013 & RG & Short & 9 & 30 & - \\
\hline Bertels and Fahle, 2006 & RG & Short & 50 & 600 & - \\
\hline Braekers et al. 2016 & $\mathrm{R}$ & Short & 89 & 300 & - \\
\hline Bredström and Rönnqvist, 2008 & RG & Short & 16 & 90 & - \\
\hline \begin{tabular}{|l|l|} 
Eveborn et al. & 2006 \\
\end{tabular} & $\mathrm{R}$ & Short & 20 & 123 & - \\
\hline Hiermann et al. 2013. & $\mathrm{R}$ & Short & 518 & 717 & - \\
\hline Issaoui et al. 2015 & $\mathrm{L}^{a}$ & Short & 4 & - & $30^{\square}$ \\
\hline Liu et al. 2013 & I间|| & Short & $50^{\square}$ & $200^{\mathbb{a}}$ & - \\
\hline $\begin{array}{lll}\text { Liu et al. } & 2017 \\
\end{array}$ & $\mathrm{R} / \mathrm{L}^{b}$ & Short & 12 & 100 & - \\
\hline Mankowska et al. 2014 & $\mathrm{R}$ & Short & 40 & - & 300 \\
\hline Rasmussen et al. 2012 & $\mathrm{R} / \mathrm{RG} / \mathrm{L}^{e}$ & Short & $15 / 15 / 16^{e}$ & $150 / 150 / 80^{\Theta}$ & - \\
\hline Redjem and Marcon, 2016 & RG & short & 30 & 60 & 240 \\
\hline Rendl et al. 2012 & $\mathrm{R}$ & Short & 518 & 717 & - \\
\hline Rest and Hirsch, 2016. & $\mathrm{R}$ & Short & 46 & 202 & - \\
\hline Trautsamwieser et al. 2011 & $\mathrm{R} / \mathrm{RG}$ & Short & $75 / 20$ & $512 / 100$ & - \\
\hline Trautsamwieser and Hirsch 2011 & $\mathrm{R} / \mathrm{RG}$ & Short & $75 / 20$ & $512 / 100$ & - \\
\hline Bard et al. 2014 & $\mathrm{RG}$ & Long & 20 & - & 200 \\
\hline Bard et al. 2013 & $\mathrm{R} / \mathrm{RG}$ & Long & $12 / 16$ & $278 /-$ & $-/ 160$ \\
\hline Begur et al. 1997 & $\mathrm{R}$ & Long & 7 & - & 200 \\
\hline Bowers et al. 2015 & $\mathrm{RG}$ & Long & 5 & - & 140 \\
\hline Bräysy et al. 2009 & $\mathrm{R}$ & Long & 9 & - & 324 \\
\hline Cappanera and Scutellà, 2015 & $\mathrm{R} / \mathrm{L}$ & Long & $11 / 11^{⿴ 囗 十 丁}$ & - & $162 / 36 丹$ \\
\hline Duque et al. 2015 & $\mathrm{R}$ & Long & 25 & - & 109 \\
\hline Hewitt et al. 2016. & $\mathrm{R}$ & Long & 75 & 400 & - \\
\hline Ikegami and Uno, 2007. & $\mathrm{R}$ & Long & 18 & 31 & - \\
\hline \begin{tabular}{|l|l|} 
Liu et al. & 2014 \\
\end{tabular} & $\mathrm{R} / \mathrm{L}^{\text {Etra }}$ & Long & $2 / 50^{\mathbb{d}}$ & $-/ 200^{0}$ & $58 /-$ \\
\hline \begin{tabular}{|l|l|} 
Nickel et al. & 2012 \\
\end{tabular} & $\mathrm{R}$ & Long & 12 & 361 & - \\
\hline Trautsamwieser and Hirsch 2014 & $\mathrm{R}$ & Long & 9 & 203 & - \\
\hline Wirnitzer et al., 2016 & $\mathrm{R}$ & Long & 37 & 143 & 1114 \\
\hline
\end{tabular}

R: Real Data, RG: Randomly Generated Data, L: Data from Literature

${ }^{a}$ Trautsamwieser et al. 2011.

$b \overline{\text { Solomon } 1987}$

$c$ Gehring and Homberger 1999

$d$ Hasama et al. 1998 .

$e$ Bredstrom and Ronnqvist 2007

$f$ Nickel et al. 2012

$g$ Jensen 2012. 

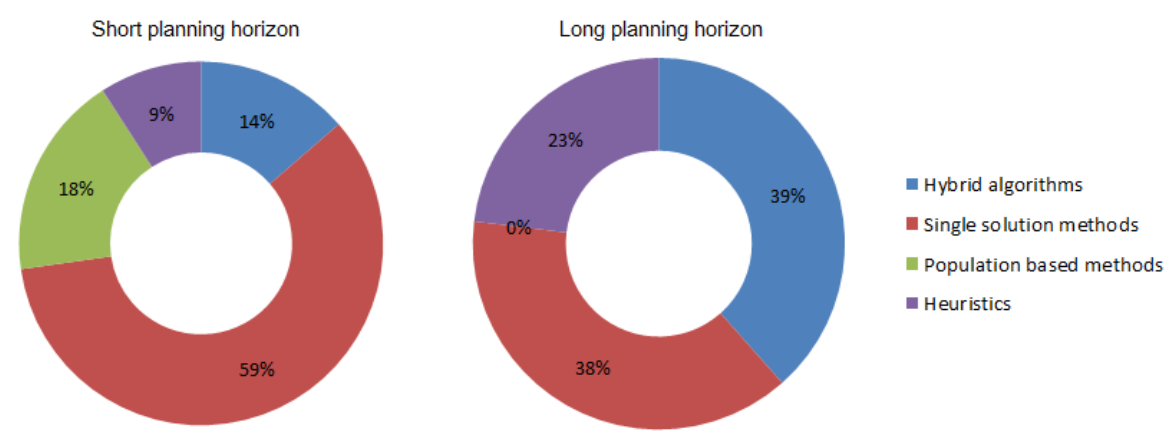

Figure 7: Publications with respect to methods

\subsection{Benchmark analysis}

Information related to the data sets are summarized in Table 8 in order to present instances that are used in different works. In this table, we also provide the information partitioning to the instance type (randomly generated (RG), real (R) and literature (L)), planning horizon (short or long), maximum number of available care workers, patients and provided services. Papers that do not specify such information are not included in the table. As seen, almost all papers have used different instances with different parametric settings. Few instances are commonly used in different papers for which at least one author of these works is the same. Thus, there are no available instances that are used or can be used for benchmark analysis.

\section{Conclusions, Research Gaps and Future Directions}

This paper reviews the recent OR models developed for the HHCRSP. For this purpose, the typical features involved in HHC organizations have been highlighted to capture the complexity associated with such systems. Hence, features may concern HHC service organization, care workers and patient characteristics. Then, a thorough literature review has been conducted that highlights how the identified features are considered in existing models, either as constraints or as criterion to be satisfied within the objective functions. Hence, three types of constraints, namely temporal, assignment and geographical constraints, have been identified for each group of features. We also classify existing papers according to solution methods they develop and the length of the planning horizon considered (single or several periods).

Our review reveals that many different modeling approaches have been proposed in the HHCRSP literature, in terms of specific topics analyzed, objectives studied, and the associated regulative contexts. The large number of models can be explained by the diversity of modeling contexts, which differ by countries and regulations, the type of structures and the types of patients cared for. One of the consequences is that methods proposed in the literature to solve these problems are difficult. Furthermore, even though multiple authors publish their benchmark instances online, experimentation is rarely based on existing instances. 
A promising future research avenue would consist of developing more generic models and flexible methods able to solve a larger number of problems. Such tools offer a great potential in terms of the HHCRSP solution improvement and cost efficiency. Indeed, some studies show the benefits of such tools. Bard et al. 2014 have reported a reduction of $18 \%$ in costs with respect to manual planning. Eveborn et al., 2006 have reported savings of $7 \%$ on the total working time and $20 \%$ on the total traveling time.

Another feature that also seems interesting to consider in future research is the multiple time windows defining all periods in which patients are available to receive care services. In most HHC contexts, patients cannot leave their homes. However, even if they are available all day to receive care services, they prefer not to be disturbed during some parts of the day, such as lunch times, nap times for elders, and so on. Taking into account the multiple time windows will make the evaluation of a route more complex because the choice of time windows to visit the patient is not simple. No classic algorithm can be used to evaluate such a route. For example, Tricoire et al. 2010 have developed a new procedure to check the route feasibility with regard to multiple time windows and route duration limits for a multi-period orienteering problem.

In terms of objective function, most research uses a weighted sum to take into account several criteria. This approach cannot obtain the entire Pareto front, especially non-supported points. Only a few recent papers deal with a multicriteria approach (Braekers et al. 2016 and Duque et al., 2015). Multi-objective resolution methods find different solutions for the decision makers of HHC structures in order to help them choose a good balance of the quality of care and the total costs, for example.

A large portion of the existing studies focus on deterministic HHCRSP models without considering the uncertain aspects related to HHC operations even if HHC providers regularly have to face this issue in practice. Different parameters involve uncertainty, such as traveling times between two different locations (especially in urban areas), care service duration, emergencies, and workers' or patients' unavailabilities. These uncertainties can be captured by stochastic models Gendreau et al. 1996. In a dynamic environment, some random events can also partially or totally reschedule the routes of care workers, such as the cancellation of appointments or last-minute absences of care workers. In this case, the routing problem is similar to dynamic vehicle routing problems Pillac et al. 2013, which have recently received particular attention. Only a few papers deal with the dynamic version of the HHCRSP Bennett and Erera, 2011, Hewitt et al., 2016. The implementation of such stochastic/dynamic models and methods can be facilitated by several technologies that are available for HHC, including global positioning system (GPS) embedded in care worker vehicles in order to determine their geographical position; smart home technologies such as sensors; Internet-enabled home monitors; cellular connectivity via mobile phones; and telemedicine as well as more sophisticated travel time modeling capabilities, e.g., the use of GIS techniques to analyze and display spatial data. Another point concerns the transportation mode considered in existing studies. Public transportion plays an important role in urban cities. Furthermore, unit transportation can be shared between workers 
that share the same shifts in a given area. Therefore, the extension of current models that consider such levers seems to be a relevant research direction.

Even if several models and methods have been proposed and other new ones will be developed for the HHCRSP literature, there are no available instance sets to directly compare these models and/or methods as explained in Section 5.3. Thus, another important future research direction would be to generate such benchmark instance sets enabling comparative analysis of models and methods.

\section{Acknowledgments}

This research was conducted as part of the PONOSAD project. The PONOSAD project is supported by The French National Research Agency (Agence Nationale de la Recherche - ANR) through grant ANR-13JS02-0001-01.

\section{References}

Akjiratikarl, C., Yenradee, P., Drake, P. R., Nov. 2007. PSO-based algorithm for home care worker scheduling in the UK. Computers \& Industrial Engineering 53 (4), 559-583.

Allaoua, H., Borne, S., Létocart, L., Wolfler Calvo, R., 2013. A matheuristic approach for solving a home health care problem. Electronic Notes in Discrete Mathematics 41, 471-478.

Amorim, P., Almada-Lobo, B., Jan. 2014. The impact of food perishability issues in the vehicle routing problem. Computers \& Industrial Engineering 67, 223-233.

Baptista, S., Oliveira, R. C., Zúquete, E., 2002. A period vehicle routing case study. European Journal of Operational Research 139 (2), 220-229.

Bard, J. F., Shao, Y., Jarrah, A. I., Apr. 2014. A sequential GRASP for the therapist routing and scheduling problem. Journal of Scheduling 17 (2), 109-133.

Bard, J. F., Shao, Y., Wang, H., Sep. 2013. Weekly scheduling models for traveling therapists. Socio-Economic Planning Sciences 47 (3), 191-204.

Bashir, B., Chabrol, M., Caux, C., 2012. Literature review in home care. Proceedings of MOSIM'12.

Begur, S. V., Miller, D. M., Weaver, J. R., Aug. 1997. An integrated spatial DSS for scheduling and routing home-health-care nurses. Interfaces 27 (4), 35-48.

Bektas, T., 2006. The multiple traveling salesman problem: An overview of formulations and solution procedures. Omega 34 (3), 209-219. 
Bennett, A. R., 2010. Home health care logistics planning. Ph.D. thesis, Georgia Institute of Technology.

Bennett, A. R., Erera, A. L., Mar. 2011. Dynamic periodic fixed appointment scheduling for home health. IIE Transactions on Healthcare Systems Engineering 1 (1), 6-19.

Bennett Milburn, A. R., 2012. Operations Research Applications in Home Healthcare. In: Hall, R. (Ed.), Handbook of Healthcare System Scheduling. Vol. 168 of International Series in Operations Research \& Management Science. Springer US, Boston, MA, Ch. 11, pp. 281-302.

Benzarti, E., 2012. Home health care operations management: Applying the districting approach to Home Health Care. Ph.D. thesis, Ecole Centrale Paris.

Benzarti, E., Sahin, E., Dallery, Y., 2010. A literature review on operations management based models developed for home health care services. Tech. rep., Ecole Centrale Paris, Paris.

Bertels, S., Fahle, T., Oct. 2006. A hybrid setup for a hybrid scenario: Combining heuristics for the home health care problem. Computers \& Operations Research 33 (10), 2866-2890.

Blais, M., Lapierre, S. D., Laporte, G., Nov. 2003. Solving a home-care districting problem in an urban setting. Journal of the Operational Research Society 54 (11), 1141-1147.

Bowers, J., Cheyne, H., Mould, G., Page, M., 2015. Continuity of care in community midwifery. Health Care Management Science 18 (2), 195-204.

Braekers, K., Hartl, R. F., Parragh, S. N., Tricoire, F., Jan. 2016. A bi-objective home care scheduling problem: Analyzing the trade-off between costs and client inconvenience. European Journal of Operational Research 248 (2), 428-443.

Bräysy, O., Arola, J., Dullaert, W., Väisänen, J., 2012. Planning strategies for home care services. NorthKarelia University of Applied Sciences.

Bräysy, O., Gendreau, M., Feb. 2005a. Vehicle Routing Problem with Time Windows, Part I: Route construction and Local Search Algorithms. Transportation Science 39 (1), 104-118.

Bräysy, O., Gendreau, M., Feb. 2005b. Vehicle Routing Problem with Time Windows, Part II: Metaheuristics. Transportation Science 39 (1), 119-139.

Bräysy, O., Nakari, P., Dullaert, W., Neittaanmäki, P., Oct. 2009. An optimization approach for communal home meal delivery service: A case study. Journal of Computational and Applied Mathematics 232 (1), $46-53$. 
Bredstrom, D., Ronnqvist, M., 2007. A Branch and Price Algorithm for the Combined Vehicle Routing and Scheduling Problem With Synchronization Constraints. NHH Dept of Finance \& Management Science Discussion Paper No 2227/7.

Bredström, D., Rönnqvist, M., Nov. 2008. Combined vehicle routing and scheduling with temporal precedence and synchronization constraints. European Journal of Operational Research 191 (1), 19-31.

Busby, C. R., Carter, M. W., 2006. A decision tool for negotiating home care funding levels in Ontario. Home Health Care Services Quarterly 25 (3-4), 91-106.

Cappanera, P., Gouveia, L., Scutellà, M. G., 2011. The skill vehicle routing problem. In: Network Optimization. Springer Berlin Heidelberg, pp. 354-364.

Cappanera, P., Scutellà, M. G., Nov. 2015. Joint assignment, scheduling, and routing models to home care optimization: A pattern-based approach. Transportation Science 49 (4), 830-852.

Chahed, S., Marcon, E., Sahin, E., Feillet, D., Dallery, Y., 2009. Exploring new operational research opportunities within the home care context: The chemotherapy at home. Health Care Management Science $12(2), 179-191$.

Cheng, E., Rich, J., 1998. A home health care routing and scheduling problem. Tech. rep., Rice University.

Cire, A. A., Hooker, J. N., 2012. A Heuristic Logic-Based Benders Method for the Home Health Care Problem. Tech. rep., Tepper School of Business.

Clarke, G., Wright, J. W., Aug. 1964. Scheduling of Vehicles from a Central Depot to a Number of Delivery Points. Operations Research $12(4), 568-581$.

Cordeau, J.-F., Gendreau, M., Laporte, G., Sep. 1997. A Tabu search heuristic for periodic and multi-depot vehicle routing problems. Networks 30 (2), 105-119.

Cordeau, J.-F., Laporte, G., Mercier, A., 2001. A unified tabu search heuristic for vehicle routing problems with time windows. Journal of the Operational Research Society 52 (8), 928-936.

Dantzig, G., Ramser, J. H., Oct. 1959. The Truck Dispatching Problem. Management Science 6 (1), 80-91.

De Angelis, V., 1998. Planning Home Assistance for AIDS Patients in the City of Rome, Italy. Interfaces $28(3), 75-83$.

Di Gaspero, L., Urli, T., 2014. A CP/LNS approach for multi-day homecare scheduling problems. In: International Workshop on Hybrid Metaheuristics. Springer International Publishing, pp. 1-15. 
Drexl, M., 2012. Synchronization in Vehicle Routing-A survey of VRPs with multiple synchronization constraints. Transportation Science 46 (3), 297-316.

Duque, P. M., Castro, M., Sörensen, K., Goos, P., 2015. Home care service planning. the case of landelijke thuiszorg. European Journal of Operational Research 243 (1), 292-301.

Elbenani, B., Ferland, J., Gascon, V., Dec. 2008. Mathematical programming approach for routing home care nurses. In: 2008 IEEE International Conference on Industrial Engineering and Engineering Management. IEEE, Singapore, pp. 107-111.

Eveborn, P., Flisberg, P., Rönnqvist, M., 2006. Laps Care - an operational system for staff planning of home care. European Journal of Operational Research 171 (3), 962-976.

Eveborn, P., Ronnqvist, M., Einarsdottir, H., Eklund, M., Liden, K., Almroth, M., 2009. Operations research improves quality and efficiency in home care. Interfaces 39 (1), 18-34.

Fikar, C., Hirsch, P., 2017. Home health care routing and scheduling: A review. Computers \& Operations Research 77, 86-95.

Francis, P., Smilovitz, K., Tzur, M., 2008. The period vehicle routing problem and its extensions. In: The Vehicle Routing Problem: Latest Advances and New Challenges. Springer US, pp. 73-102.

Freeman, G., Hughes, J., 2010. Continuity of Care and the Patient Experience. London: The King's Fund.

Gamst, M., Jensen, T. S., 2012. A branch-and-price algorithm for the long-term home care scheduling problem. In: Operations Research Proceedings 2011. Springer Berlin Heidelberg, pp. 483-488.

Gehring, H., Homberger, J., Jan. 1999. A parallel hybrid evolutionary metaheuristic for the vehicle routing problem with time windows. In: Proceedings of EUROGEN99. Vol. 2. pp. 57-64.

Gendreau, M., Laporte, G., Séguin, R., Jan. 1996. Stochastic vehicle routing. European Journal of Operational Research 88 (1), 3-12.

Genet, N., Boerma, W., Kroneman, M., Hutchinson, A., Saltman, R. B., 2013. Home care across europe. London: European Observatory on Health Systems and Policies.

Groër, C., Golden, B., Wasil, E., Oct. 2009. The consistent vehicle routing problem. Manufacturing \& Service Operations Management 11 (4), 630-643.

Gutiérrez, E. V., Vidal, C. J., 2013. Home health care logistics management problems: A critical review of models and methods. Revista Facultad de Ingeniería Universidad de Antioquia (68), 160-175. 
Hasama, T., Kokubugata, H., Kawashima, H., 1998. A heuristic approach based on the string model to solve vehicle routing problem with backhauls. In: Towards the New Horizon Together. Proceedings of the 5th World Congress on Intelligent Transport Systems (ITS). No. 3052. Seoul, Korea.

Hewitt, M., Nowak, M., Nataraj, N., 2016. Planning Strategies for Home Health Care Delivery. Asia-Pacific Journal of Operational Research 33 (5).

Hiermann, G., Prandtstetter, M., Rendl, A., Puchinger, J., Raidl, G. R., 2013. Metaheuristics for solving a multimodal home-healthcare scheduling problem. Central European Journal of Operations Research $23(1), 89-113$.

Ikegami, A., Uno, A., 2007. Bounds for staff size in home help staff scheduling. Journal of the Operations Research Society of Japan 50 (4), 563-575.

Issaoui, B., Zidi, I., Marcon, E., Ghedira, K., 2015. New multi-objective approach for the home care service problem based on scheduling algorithms and variable neighborhood descent. Electronic Notes in Discrete Mathematics 47, 181-188.

Jensen, T., 2012. Application of Metaheuristics to Real-Life Scheduling Problems. Ph.d. thesis, University of Southern Denmark.

Kergosien, Y., Lenté, C., Billaut, J.-c., 2009. Home health care problem: An extended multiple traveling salesman problem. In: 4th Multidisciplinary International Conference on Scheduling : Theory and Applications (MISTA'09). Dublin, Ireland, pp. 10-12.

Kergosien, Y., Ruiz, A., Soriano, P., 2014. Solving a routing problem for medical test sample collection in home health care. In: Proceedings of the International Conference on Health Care Systems Engineering. Springer International Publishing, pp. 29-46.

Kovacs, A. A., Golden, B. L., Hartl, R. F., Parragh, S. N., 2014. The generalized consistent vehicle routing problem. Transportation Science 49 (4), 796-816.

Lanzarone, E., Matta, A., Sahin, E., 2012. Operations management applied to home care services: The problem of assigning human resources to patients. IEEE Transactions on Systems, Man, and Cybernetics Part A:Systems and Humans 42 (6), 1346-1363.

Laporte, G., 2007. What You Should Know about the Vehicle Routing Problem. Naval Research Logistics (NRL) $54(8), 811-819$.

Liu, R., Xie, X., Augusto, V., Rodriguez, C., 2013. Heuristic algorithms for a vehicle routing problem with simultaneous delivery and pickup and time windows in home health care. European Journal of Operational Research 230 (3), 475-486. 
Liu, R., Xie, X., Garaix, T., 2014. Hybridization of Tabu Search with Feasible and Infeasible Local Searches for Periodic Home Health Care Logistics. Omega 47, 17-32.

Liu, R., Yuan, B., Jiang, Z., 2017. Mathematical model and exact algorithm for the home care worker scheduling and routing problem with lunch break requirements. International Journal of Production Research $55(2), 558-575$.

Macdonald, T., Dörner, K., Gandibleux, X., 2009. Metaheuristics for the consistent nurse scheduling and routing problem. Master's thesis, Université de Nantes.

Mankowska, D. S., Meisel, F., Bierwirth, C., 2014. The home health care routing and scheduling problem with interdependent services. Health Care Management Science 17 (1), 15-30.

Matta, A., Chahed, S., Sahin, E., Dallery, Y., 2014. Modelling home care organisations from an operations management perspective. Flexible Services and Manufacturing Journal 26 (3), 295-319.

Mutingi, M., Mbohwa, C., Dec. 2013. Home healthcare staff scheduling: A taxonomic state-of-the-art review. In: 2013 IEEE International Conference on Industrial Engineering and Engineering Management. pp. $1107-1111$.

Nickel, S., Schröder, M., Steeg, J., 2012. Mid-term and short-term planning support for home health care services. European Journal of Operational Research 219 (3), 574-587.

Pillac, V., Gendreau, M., Guéret, C., Medaglia, A. L., 2013. A review of dynamic vehicle routing problems. European Journal of Operational Research 225 (1), 1-11.

Pisinger, D., Ropke, S., 2007a. A general heuristic for vehicle routing problems. Computers \& Operations Research 34 (8), 2403-2435.

Pisinger, D., Ropke, S., 2007b. A general heuristic for vehicle routing problems. Computers \& Operations Research 34 (8), 2403-2435.

Prins, C., 2004. A simple and effective evolutionary algorithm for the vehicle routing problem. Computers \& Operations Research 31 (12), 1985-2002.

Quttineh, N.-H., Larsson, T., Lundberg, K., Holmberg, K., 2013. Military aircraft mission planning: A generalized vehicle routing model with synchronization and precedence. EURO Journal on Transportation and Logistics 2 (1-2), 109-127.

Rasmussen, M. S., Justesen, T., Dohn, A., Larsen, J., Jun. 2012. The Home Care Crew Scheduling Problem: Preference-based visit clustering and temporal dependencies. European Journal of Operational Research 219 (3), 598-610. 
Redjem, R., Marcon, E., 2016. Operations management in the home care services: A heuristic for the caregivers' routing problem. Flexible Services and Manufacturing Journal 28 (1-2), 280-303.

Reinhardt, L. B., Clausen, T., Pisinger, D., 2013. Synchronized dial-a-ride transportation of disabled passengers at airports. European Journal of Operational Research 225 (1), 106-117.

Rendl, A., Prandtstetter, M., Hiermann, G., Puchinger, J., Raidl, G., 2012. Hybrid heuristics for multimodal homecare scheduling. In: International Conference on Integration of Artificial Intelligence (AI) and Operations Research (OR) Techniques in Contraint Programming. Springer Berlin Heidelberg, pp. $339-355$.

Rest, K.-d., Hirsch, P., 2016. Daily scheduling of home health care services using time-dependent public transport. Flexible Services and Manufacturing Journal 28 (3), 495-525.

Rest, K.-D., Trautsamwieser, A., Hirsch, P., 2012. Trends and risks in home health care. Journal of Humanitarian Logistics and Supply Chain Management 2 (1), 34-53.

Rintala, A., Karppinen, H., Koivuniemi, J., 2016. Operations management in improving elderly home care. International Journal of Services and Operations Management 24 (3), 331-362.

Russell, R., 1995. Hybrid heuristics for the vehicle routing problem with time windows. Transportation Science 29 (2), 156-166.

Sahin, E., Matta, A., 2015. A contribution to operations management-related issues and models for home care structures. International Journal of Logistics Research and Applications 18 (4), 355-385.

Savelsbergh, M. W., 1992. The vehicle routing problem with time windows: Minimizing route duration. ORSA Journal on Computing 4 (2), 146-154.

Shao, Y., 2011. Home Therapist Network Modeling. Ph.D. thesis, University of Texas at Austin.

Solomon, M., 1987. Algorithms for the vehicle routing and scheduling problems with time window constraints. Operations Research 35 (2), 254-265.

Steeg, J., Schröder, M., 2008. A Hybrid approach to solve the periodic home health care problem. In: Operations Research Proceedings 2007. Springer Berlin Heidelberg, pp. 297-302.

Thomsen, K., 2006. Optimization on Home Care. Master's thesis, Technical University of Denmark.

Toth, P., Vigo, D. (Eds.), 2001. The vehicle routing problem. Siam Monographs on Discrete Mathematics and Applications. 
Trautsamwieser, A., Gronalt, M., Hirsch, P., 2011. Securing home health care in times of natural disasters. OR Spectrum $33(3), 787-813$.

Trautsamwieser, A., Hirsch, P., 2011. Optimization of daily scheduling for home health care services. Journal of Applied Operational Research 3 (3), 124-136.

Trautsamwieser, A., Hirsch, P., 2014. A branch-price-and-vut approach for solving the medium-term home health care planning problem. Networks 64 (3), 143-159.

Tricoire, F., 2012. Multi-directional local search. Computers \& Operations Research 39 (12), 3089-3101.

Tricoire, F., Romauch, M., Doerner, K. F., Hartl, R. F., 2010. Heuristics for the multi-period orienteering problem with multiple time windows. Computers \& Operations Research 37 (2), 351-367.

Vansteenwegen, P., Souffriau, W., Van Oudheusden, D., 2011. The orienteering problem: A survey. European Journal of Operational Research 209 (1), 1-10.

Vidal, T., Crainic, T. G., Gendreau, M., Prins, C., 2013a. A hybrid genetic algorithm with adaptive diversity management for a large class of vehicle routing problems with time-windows. Computers \& Operations Research 40 (1), 475-489.

Vidal, T., Crainic, T. G., Gendreau, M., Prins, C., 2013b. Heuristics for multi-attribute vehicle routing problems: A survey and synthesis. European Journal of Operational Research 231 (1), 1-21.

Wang, H., 2011. The Therapist Scheduling Problem for Patients with Fixed Appointment Times. Master's thesis.

Wirnitzer, J., Heckmann, I., Meyer, A., Nickel, S., 2016. Patient-based nurse rostering in home care. Operations Research for Health Care 8, 91-102.

Yalcindag, S., Cappanera, P., Grazia Scutella, M., Sahin, E., Matta, A., 2016a. Pattern-based decompositions for human resource planning in home health care services. Computers and Operations Research 73, $12-26$.

Yalcindag, S., Matta, A., Sahin, E., 2012a. Human Resource Scheduling and Routing Problems in Home Health Care Context: A Literature Review. Tech. rep., Ecole Centrale Paris, Paris.

Yalcindag, S., Matta, A., Sahin, E., Aug. 2012b. Operator assignment and routing problems in home health care services. In: 2012 IEEE International Conference on Automation Science and Engineering (CASE). IEEE, Seoul, pp. 329-334.

Yalcindag, S., Matta, A., Sahin, E., Shanthikumar, J. G., 2016b. The patient assignment problem in home health care: Using a data-driven method to estimate the travel times of care givers. Flexible Services and Manufacturing Journal 28 (1-2), 304-335. 